\title{
The quasi-geostrophic ellipsoidal vortex model
}

\author{
By DAVID G. DRITSCHEL, JEAN N. REINAUD \\ AND WILLIAM J. MCKIVER
}

School of Mathematics and Statistics, University of St Andrews, St Andrews, UK

(Received 18 February 2003 and in revised form 17 July 2003)

We present a simple approximate model for studying general aspects of vortex interactions in a rotating stably-stratified fluid. The model idealizes vortices by ellipsoidal volumes of uniform potential vorticity, a materially conserved quantity in an inviscid, adiabatic fluid. Each vortex thus possesses 9 degrees of freedom, 3 for the centroid and 6 for the shape and orientation. Here, we develop equations for the time evolution of these quantities for a general system of interacting vortices. An isolated ellipsoidal vortex is well known to remain ellipsoidal in a fluid with constant background rotation and uniform stratification, as considered here. However, the interaction between any two ellipsoids in general induces weak non-ellipsoidal perturbations. We develop a unique projection method, which follows directly from the Hamiltonian structure of the system, that effectively retains just the part of the interaction which preserves ellipsoidal shapes. This method does not use a moment expansion, e.g. local expansions of the flow in a Taylor series. It is in fact more general, and consequently more accurate. Comparisons of the new model with the full equations of motion prove remarkably close.

\section{Introduction}

Vortex interactions are an integral part of the evolution of high-Reynolds-number fluid flows. In rotating stably-stratified flows such as the atmosphere and the oceans, vortex interactions often generate larger vortices (as well as smaller fragments and filaments), e.g. through vortex merger (cf. McWilliams, Weiss \& Yavneh 1999; Dritschel 2002; Reinaud \& Dritschel 2002, and references therein). Indeed, it is principally through such vortex interactions that these flows evolve.

However, vortex interactions are fundamentally nonlinear, and often exceedingly complex. Little is known analytically, and numerical simulations are too costly to obtain any comprehensive understanding of the mechanisms involved. The basic parameter space is huge, and a direct attack is out of the question (cf. Reinaud \& Dritschel 2003).

Here, we present an alternative approximate way forward. The starting point is the quasi-geostrophic (QG) equations, themselves approximations of the full primitive equations governing rotating stratified flows (see Simmons \& Hoskins 1976; Gill 1982; Holton 1982, and many others). The QG equations have been widely used in this context, and capture the main features of these vortically-driven flows (see, e.g. Dritschel \& Viúdez 2003 and references therein). In the QG system, the flow is driven by a single scalar field called the 'potential vorticity' (PV, see Hoskins, McIntyre \& Robertson 1985) which is a materially conserved scalar in the adiabatic inviscid limit considered here (a relevant limit in the present context). We also consider uniform 
constant values of the Coriolis and buoyancy frequencies, $f$ and $N$, as in previous works, and assume the flow is unbounded. These approximations simplify the equations to PV advection

$$
\frac{\mathrm{D} q}{\mathrm{D} t}=q_{t}+u q_{x}+v q_{y}=0,
$$

(here and below, the subscripts $x, y, z$ and $t$ denote partial derivatives) where $q$ is the $\mathrm{PV}$, together with a simple linear inversion

$$
\nabla^{2} \psi=q
$$

for a scalar streamfunction $\psi$, from which the flow field is obtained by

$$
\boldsymbol{u}(\boldsymbol{x}, t)=(u, v, w)=\left(-\psi_{y}, \psi_{x}, 0\right)
$$

- note the lack of vertical motion at this order (first order in Rossby number). In (1.2), $\nabla^{2}$ is the three-dimensional Laplacian. The above equations are written in a coordinate system in which the height $z$ has been stretched by $N / f$. It is through (1.2) that the system is coupled in $z$. Note the structural similarity with the two-dimensional Euler equations written in vorticity-streamfunction form.

These equations still have infinite degrees of freedom, and are nonlinear; a further simplification is needed. The simplification exploits an exact class of solutions, consisting of ellipsoids of uniform PV, discovered in the geophysical context by Zhmur \& Shchepetkin (1991) and Meacham (1992), and closely paralleling the two-dimensional elliptical solutions discovered by Kida (1981). In fact, much of the mathematical basis of these ellipsoidal solutions, excepting (1.3) which is specific to QG theory, stems from much earlier studies, principally in the eighteenth century, of self-gravitating ellipsoidal masses, culminating in the work of Chandrasekhar (1969). Recently, these ellipsoidal solutions have been studied by numerous authors, cf. Meacham et al. (1994), Meacham, Morrison \& Flierl (1997), Miyazaki, Ueno \& Shimonishi (1999), Hashimoto, Shimonishi \& Miyazaki (1999), and McKiver \& Dritschel (2003).

While a single ellipsoid in a linear background flow is an exact time-dependent solution of (1.1)-(1.3), multiple ellipsoids are not. The flow field outside one ellipsoid does not have the correct form to ensure that another ellipsoid will remain precisely ellipsoidal. An approximation must be made, and the common one made is to use a truncated moment expansion of this flow field, together with a local Taylor-series expansion of this truncated flow field about the centre of the 'target' vortex - in effect a double truncation (see Zhmur \& Pankratov 1990, and for more background, Miyazaki, Furuichi \& Takahashi 2001). This model again parallels the earlier work by Melander, Zabusky \& Styczek (1986) in the two-dimensional context.

Here, we take a different approach that avoids moment expansions altogether and arrives at a more accurate model. This approach again has two-dimensional roots in the 'elliptical model' (Legras \& Dritschel 1991). That model retains the entire part of vortex interactions which preserve the elliptical shape of vortices, and uses the exact form of the vortex-interaction energy. The 'ellipsoidal model' introduced here is developed in the same way. The equations are derived with alarming simplicity via a Hamiltonian approach, which unifies the self-induced vortex motion and the externally induced vortex motion, whether it be due to another vortex or to a linear background flow.

There is just one snag: as in two dimensions, the vortex interaction energy cannot be evaluated in closed form (it generally involves spatial integrals over elliptical functions). As in two-dimensions, we overcome this obstacle by introducing a discrete 
approximation: we use a set of singular point vortices to compute the interaction energy efficiently (in two dimensions, we can show that this is equivalent to Gaussian quadrature, a very accurate means of integration, see Dritschel \& Legras 1991). The results presented below exhibit rapid convergence with the number of point vortices, and in practice 7 point vortices are sufficiently accurate. Moreover, we can show that the moment-based models follow by further approximating the discrete interaction energy used here in the ellipsoidal model (details given in Appendix C).

In the next section, the ellipsoidal model is derived. Then, in $\S 3$ a discrete approximation is introduced for evaluating the Hamiltonian, and the discrete equations are given. A numerical algorithm is outlined in $\S 4$, then the instantaneous and long-term accuracy of the ellipsoidal model is assessed by comparing it with the full quasigeostrophic system. Some current applications of this model to the study of vortex interactions are described in $\S 5$, ending with some conclusions.

\section{Mathematical formulation of the model}

In the two-dimensional elliptical and three-dimensional ellipsoidal models, the idea is to sacrifice the detailed structure of each vortex in order to better understand their general properties, under much more wide-ranging conditions than can be feasibly considered by a direct approach. While destructive vortex interactions, such as vortex merger, cannot be modelled this way, many other important aspects of vortex interactions, such as the conditions leading to destructive interactions, can be (Reinaud \& Dritschel 2003).

Unique to our model is the use of just one assumption in deriving it: each vortex, considered to have uniform PV, remains ellipsoidal for all time. In reality, vortex interactions induce non-ellipsoidal disturbances, so our assumption implies that we are disregarding a part of the dynamics, hopefully a small part. On the other hand, the self-induced flow of an ellipsoidal vortex keeps it ellipsoidal (merely rotating it), and moreover immersing an ellipsoidal vortex in a linear background flow also keeps it ellipsoidal (though now it may deform in a complicated way).

Here we will follow the matrix representation introduced recently by McKiver \& Dritschel (2003). Each ellipsoid of uniform PV, $q$, is represented by a symmetric matrix $\mathscr{B}$ and a centre $\boldsymbol{X}$, in terms of which the surface of the ellipsoid is expressed by the set of points $\boldsymbol{x}$ satisfying

$$
(\boldsymbol{x}-\boldsymbol{X})^{T} \mathscr{B}^{-1}(\boldsymbol{x}-\boldsymbol{X})=1,
$$

where the superscript $T$ denotes transpose. The eigenvalues of $\mathscr{B}$ are the squared semi-axis lengths $a^{2}, b^{2}$ and $c^{2}$, and the eigenvectors are unit vectors $\hat{\boldsymbol{a}}, \hat{\boldsymbol{b}}$ and $\hat{\boldsymbol{c}}$ pointing along the corresponding axes. Writing

$$
\mathscr{M}=(\hat{\boldsymbol{a}} \hat{\boldsymbol{b}} \hat{\boldsymbol{c}}),
$$

we have $\mathscr{M}^{-1}=\mathscr{M}^{T}$, and the useful decomposition $\mathscr{B}=\mathscr{M} \mathscr{E} \mathscr{M}^{T}$, where $\mathscr{E}$ is the diagonal matrix of eigenvalues.

We wish to consider several interacting vortices, but for the moment let us suppose the velocity field $\boldsymbol{u}$ at the surface of the present ellipsoid $\{q, \boldsymbol{X}, \mathscr{B}\}$ is given, and moreover that it is linear in $x$ :

$$
\boldsymbol{u}(\boldsymbol{x}, t)=\boldsymbol{U}(t)+\mathscr{S}(t)(\boldsymbol{x}-\boldsymbol{X}(t)),
$$

where $\boldsymbol{U}(t)$ is an arbitrary vector and $\mathscr{S}(t)$ is an arbitrary matrix, called the 'flow matrix'. This velocity field is of the most general form which exactly keeps the vortex 
ellipsoidal. The ellipsoid evolves according to

$$
\begin{aligned}
\boldsymbol{X}_{t} & =\boldsymbol{U}, \\
\mathscr{B}_{t} & =\mathscr{S} \mathscr{B}+\mathscr{B} \mathscr{S}^{T},
\end{aligned}
$$

(see McKiver \& Dritschel 2003). Next, we employ the specific properties of the QG system. The QG velocity field must satisfy (1.3), which can also be written as

$$
\boldsymbol{u}=\mathscr{L} \nabla \psi \quad \text { or } \quad \boldsymbol{u}=\mathscr{L} \frac{\partial \psi}{\partial \boldsymbol{x}} \quad \text { with } \quad \mathscr{L}=\left(\begin{array}{ccc}
0 & -1 & 0 \\
1 & 0 & 0 \\
0 & 0 & 0
\end{array}\right) .
$$

Therefore, the most general linear QG velocity field must derive from a streamfunction of the form

$$
\psi(\boldsymbol{x}, t)=\boldsymbol{F}(t) \cdot \boldsymbol{x}+\frac{1}{2} \boldsymbol{x}^{T} \mathscr{P}(t) \boldsymbol{x},
$$

with $\boldsymbol{F}$ and $\mathscr{P}$ related to $\boldsymbol{U}$ and $\mathscr{S}$ in (2.3) by

$$
\begin{aligned}
\boldsymbol{U} & =\mathscr{L} \boldsymbol{F}+\mathscr{S} \boldsymbol{X}=\mathscr{L}(\boldsymbol{F}+\mathscr{P} \boldsymbol{X}), \\
\mathscr{S} & =\mathscr{L} \mathscr{P},
\end{aligned}
$$

- in particular, there is no vertical motion $\left(Z_{t}=0\right)$, the bottom row of $\mathscr{S}$ is zero, and $\operatorname{tr} \mathscr{S}=\mathscr{S}_{11}+\mathscr{S}_{22}=0$ (since the flow is non-divergent at each height).

We next use these results to show how the ellipsoidal vortex evolution equations (2.4) may be derived from a Hamiltonian formalism. In the full QG equations, the Hamiltonian $H$ is proportional to the total energy $E$, given by

$$
E=\frac{1}{2} \iiint|\nabla \psi|^{2} \mathrm{~d} V
$$

(ignoring the infinite energy of any background flow of the form (2.6)). We will adopt this form also for the ellipsoidal model. In particular, we take

$$
H=\frac{1}{4 \pi} E
$$

for convenience. Let us now split $\psi$ in (2.8) into a self-induced part $\psi_{v}$ and a background part $\psi_{b}$ of the form (2.6), with $\boldsymbol{F}=\boldsymbol{F}_{b}$ and $\mathscr{S}=\mathscr{S}_{b}$. Ignoring the infinite energy of the background flow, we may write

$$
H=H_{i}+H_{v},
$$

where $H_{i}$ is the part due to the interaction between the background flow and the vortex,

$$
H_{i}=\frac{1}{4 \pi} \iiint \nabla \psi_{v} \cdot \nabla \psi_{b} \mathrm{~d} V=-\frac{1}{4 \pi} \iiint_{V} q \psi_{b} \mathrm{~d} V,
$$

(using $q=\nabla^{2} \psi_{v}$ ) and $H_{v}$ is the self-induced part,

$$
H_{v}=\frac{1}{8 \pi} \iiint\left|\nabla \psi_{v}\right|^{2} \mathrm{~d} V=-\frac{1}{8 \pi} \iiint_{V} q \psi_{v} \mathrm{~d} V
$$

(the subscript $V$ denotes integration over the vortex only).

Let us first consider the interaction term $H_{i}$. Substituting in the form of $\psi_{b}$ from (2.6) (with subscripts $b$ on $\boldsymbol{F}$ and $\mathscr{S}$ ), we may readily evaluate $H_{i}$ as

$$
H_{i}=-\kappa\left(\boldsymbol{F}_{b} \cdot \boldsymbol{X}+\frac{1}{2} \boldsymbol{X}^{T} \mathscr{P}_{b} \boldsymbol{X}\right)-\frac{1}{10} \kappa \mathscr{P}_{b}: \mathscr{B},
$$


where $\kappa \equiv q V / 4 \pi=q a b c / 3$ is the vortex 'strength', where $\mathscr{P}_{b}: \mathscr{B} \equiv \sum_{j} \sum_{k} \mathscr{P}_{b j k} \mathscr{B}_{j k}$ denotes the scalar product of the two matrices, and where we have used the fact that

$$
\mathscr{B}=\frac{5}{V} \iiint_{V}(\boldsymbol{x}-\boldsymbol{X})(\boldsymbol{x}-\boldsymbol{X})^{T} \mathrm{~d} V .
$$

It follows that

$$
\begin{aligned}
\frac{\partial H_{i}}{\partial \boldsymbol{X}} & =-\kappa\left(\boldsymbol{F}_{b}+\mathscr{P}_{b} \boldsymbol{X}\right), \\
\frac{\partial H_{i}}{\partial \mathscr{B}} & =-\frac{1}{10} \kappa \mathscr{P}_{b},
\end{aligned}
$$

and comparing with (2.7), we see that

$$
\begin{aligned}
\boldsymbol{U}_{b} & =-\frac{1}{\kappa} \mathscr{L} \frac{\partial H_{i}}{\partial \boldsymbol{X}}, \\
\mathscr{S}_{b} & =-\frac{10}{\kappa} \mathscr{L} \frac{\partial H_{i}}{\partial \mathscr{B}},
\end{aligned}
$$

in (2.4). Note in particular that the background streamfunction matrix is given by

$$
\mathscr{P}_{b}=-\frac{10}{\kappa} \frac{\partial H_{i}}{\partial \mathscr{B}} .
$$

Next consider the self-induced part of the Hamiltonian, $H_{v}$. The streamfunction inside the vortex takes the form (Laplace 1784)

$$
\psi_{v}=C+\frac{1}{2}(\boldsymbol{x}-\boldsymbol{X})^{T} \mathscr{P}_{v}(\boldsymbol{x}-\boldsymbol{X}),
$$

where $\mathscr{P}_{v}=\mathscr{M} \mathscr{D} \mathscr{M}^{T}$ and $\mathscr{D}$ is a diagonal matrix with

$$
\begin{aligned}
& \mathscr{D}_{11}=\kappa R_{D}\left(b^{2}, c^{2}, a^{2}\right), \\
& \mathscr{D}_{22}=\kappa R_{D}\left(c^{2}, a^{2}, b^{2}\right), \\
& \mathscr{D}_{33}=\kappa R_{D}\left(a^{2}, b^{2}, c^{2}\right),
\end{aligned}
$$

$-R_{D}$ being the elliptic integral of the second kind - and where $C$ is given by

$$
C=-\frac{3}{2} \kappa R_{F}\left(a^{2}, b^{2}, c^{2}\right)
$$

$-R_{F}$ being the elliptic integral of the first kind (see Appendix A). This constant is required to match $\psi_{v}$ at the boundary of the ellipsoid with the decaying outer solution, which tends to $\psi_{v}=-\kappa / r$ as $r=|\boldsymbol{x}-\boldsymbol{X}| \rightarrow \infty$. Note that the inner solution is exactly quadratic, so once again the Hamiltonian $H_{v}$ (2.12) can be readily evaluated:

$$
\begin{aligned}
H_{v} & =\frac{3}{4} \kappa^{2} R_{F}\left(a^{2}, b^{2}, c^{2}\right)-\frac{1}{20} \kappa \mathscr{P}_{v}: \mathscr{B}, \\
& =\frac{3}{4} \kappa^{2} R_{F}\left(a^{2}, b^{2}, c^{2}\right)-\frac{1}{20} \kappa \mathscr{D}: \mathscr{E}, \\
& =\frac{3}{5} \kappa^{2} R_{F}\left(a^{2}, b^{2}, c^{2}\right),
\end{aligned}
$$

using $a^{2} R_{D}\left(b^{2}, c^{2}, a^{2}\right)+b^{2} R_{D}\left(c^{2}, a^{2}, b^{2}\right)+c^{2} R_{D}\left(a^{2}, b^{2}, c^{2}\right)=3 R_{F}\left(a^{2}, b^{2}, c^{2}\right)$ in the last line. Now, by direct calculation (see Appendix A), we may show that

$$
\mathscr{P}_{v}=-\frac{10}{\kappa} \frac{\partial H_{v}}{\partial \mathscr{B}},
$$

which is identical in form to (2.17), and can be inferred from the Hamiltonian analysis of Meacham et al. (1997), whereas $\partial H_{v} / \partial X=0$, confirming that there is no selfinduced motion of the vortex centre $\left(\boldsymbol{U}_{v}=0\right)$. Therefore, the self-induced vortex 
motion is obtained from the Hamiltonian in precisely the same way as the backgroundinduced motion.

This is no accident. No other relationships are consistent with the required Hamiltonian structure of the ellipsoidal model. The model - for any number of vortices - must satisfy the basic evolution equations (2.4) together with

$$
\begin{aligned}
\boldsymbol{U} & =-\frac{1}{\kappa} \mathscr{L} \frac{\partial H}{\partial \boldsymbol{X}}, \\
\mathscr{S} & =-\frac{10}{\kappa} \mathscr{L} \frac{\partial H}{\partial \mathscr{B}},
\end{aligned}
$$

for each vortex. Here, $H$ is the full Hamiltonian, including all vortex-interaction terms (analogous to $H_{i}$ above). That is, there is a single framework for all aspects of the model.

The form of $H_{i}$ for a pair of vortices $\{q, \boldsymbol{X}, \mathscr{B}\}$ and $\left\{q^{\prime}, \boldsymbol{X}^{\prime}, \mathscr{B}\right\}$ is

$$
\begin{aligned}
H_{i} & =\frac{1}{4 \pi} \iiint \nabla \psi_{v} \cdot \nabla \psi_{v}^{\prime} \mathrm{d} V \\
& =-\frac{1}{4 \pi} \iiint_{V} q \psi_{v}^{\prime} \mathrm{d} V=-\frac{1}{4 \pi} \iiint_{V^{\prime}} q^{\prime} \psi_{v} \mathrm{~d} V^{\prime} \\
& =\frac{q^{\prime} q}{16 \pi^{2}} \iiint_{V^{\prime}} \mathrm{d} V^{\prime} \iiint_{V} \mathrm{~d} V \frac{1}{\left|\boldsymbol{x}^{\prime}-\boldsymbol{x}\right|},
\end{aligned}
$$

using the Green function integral form of the streamfunction, i.e.

$$
\psi_{v}\left(\boldsymbol{x}^{\prime}\right)=-\frac{q}{4 \pi} \iiint_{V} \frac{\mathrm{d} V}{\left|\boldsymbol{x}^{\prime}-\boldsymbol{x}\right|} .
$$

In these expressions, the volume integrals are over the respective ellipsoids only. Now, however, $H_{i}$ cannot be evaluated in closed form, and recourse must be made to a discrete approximation (outlined in the following section); but whatever approximation is used, the ellipsoidal motion induced by $H_{i}$ is still given by (2.16).

\section{The discrete ellipsoidal model}

We next approximate $H_{i}$ in a novel way by seeking an efficient numerical quadrature formula which accurately approximates the volume integrals. This was previously done in the two-dimensional elliptical model (Legras \& Dritschel 1991), where Gaussian quadrature along the line connecting the foci of each interacting ellipse was found to be optimal.

By analogy with the two-dimensional problem, we expect that the quadrature formula can be obtained by approximating the external streamfunction $\psi_{v}$ of an ellipsoidal vortex by that due to a discrete set of appropriately chosen singular point vortices, i.e.

$$
\psi_{v}\left(\boldsymbol{x}^{\prime}\right)=-\sum_{j=1}^{n} \frac{\kappa_{j}}{\left|\boldsymbol{x}^{\prime}-\boldsymbol{x}_{j}\right|},
$$

where the $\boldsymbol{x}_{j}$ are their locations and the $\kappa_{j}$ are their strengths. The problem is to find the $\boldsymbol{x}_{j}$ and the $\kappa_{j}$, for given $n$, which best approximates (2.25) outside of the ellipsoid.

In particular, we demand that the far-field behaviour of $\psi_{v}$ be as accurate as possible. As $r=\left|\boldsymbol{x}^{\prime}-\boldsymbol{X}\right| \rightarrow \infty$, we may show that

$$
\psi_{v}\left(\boldsymbol{x}^{\prime}\right)=-\kappa r^{-1}+M_{3}(\phi, \lambda) r^{-3}+M_{5}(\phi, \lambda) r^{-5}+O\left(r^{-7}\right),
$$


where $\phi$ and $\lambda$ are co-latitude and longitude variables defined by $\boldsymbol{x}^{\prime}-\boldsymbol{X}=r(\sin \phi \cos \lambda$, $\sin \phi \sin \lambda, \cos \phi)$ and $M_{3}, M_{5}$, etc., are known functions depending also on the shape of the ellipsoid (details omitted). In the two-dimensional problem, the analogous expansion was shown to be equivalent to that of a certain vortex sheet (a line of infinite PV) connecting the two foci of the ellipse (Legras \& Dritschel 1991). This meant that the exterior flow could be approximated simply by discretizing this sheet into a finite number of point vortices. In the three-dimensional problem here, the analogue is a two-dimensional elliptical sheet confined to the middle-major axis plane (spanned by the unit vectors $\hat{\boldsymbol{b}}$ and $\hat{\boldsymbol{c}}$ ), i.e.

$$
\varrho^{2}=\frac{\tilde{y}^{2}}{b^{2}-a^{2}}+\frac{\tilde{z}^{2}}{c^{2}-a^{2}}<1 \quad(a \leqslant b \leqslant c),
$$

where $\tilde{y}=\hat{\boldsymbol{b}} \cdot(\boldsymbol{x}-\boldsymbol{X})$ and $\tilde{z}=\hat{\boldsymbol{c}} \cdot(\boldsymbol{x}-\boldsymbol{X})$. Moreover, the PV density of the sheet is

$$
\sigma(\varrho)=\frac{6 \kappa}{\eta \tau} \sqrt{1-\varrho^{2}}
$$

where $\eta^{2}=b^{2}-a^{2}$ and $\tau^{2}=c^{2}-a^{2}$ (this result may be derived from a theorem originally due to Maclaurin (1742) and extended by Laplace (1784) to three unequal axis lengths; see p. 49 of Chandrasekhar (1969) and Appendix B for further details). This sheet is known as the 'focal ellipse' (Khavinson \& Shapiro 1989), and it is the focal ellipse for an entire 'confocal family' of ellipsoids spanned by a parameter $\chi$ :

$$
\frac{\tilde{x}^{2}}{a^{2}+\chi}+\frac{\tilde{y}^{2}}{b^{2}+\chi}+\frac{\tilde{z}^{2}}{c^{2}+\chi} \leqslant 1 \quad\left(-a^{2} \leqslant \chi<\infty\right),
$$

where $\tilde{x}=\hat{\boldsymbol{a}} \cdot(\boldsymbol{x}-\boldsymbol{X})$. All members of this family with $-a^{2} \leqslant \chi<0$ induce the same flow field outside the original ellipsoid with $\chi=0$, if we scale the PV by the factor $a b c / \sqrt{\left(a^{2}+\chi\right)\left(b^{2}+\chi\right)\left(c^{2}+\chi\right)}$ so as to keep $\kappa$ invariant (this is Maclaurin's theorem).

Thus, by analogy with the two-dimensional problem, the locations of the point vortices $\boldsymbol{x}_{j}$ and their strengths $\kappa_{j}, j=1,2, \ldots, n$, may be determined simply by matching the spatial moments of the focal ellipse up to a certain order. These moments involve only even powers of $\tilde{y}$ and $\tilde{z}$ due to symmetry; they are defined by

$$
\begin{aligned}
\mu_{k, \ell} & =\frac{1}{4 \pi} \iint \sigma(\varrho) \tilde{y}^{2 k} \tilde{z}^{2 \ell} \mathrm{d} \tilde{y} \mathrm{~d} \tilde{z} \\
& \equiv \kappa \eta^{2 k} \tau^{2 \ell} W_{k+\ell} \Theta_{k, \ell},
\end{aligned}
$$

where

$$
\begin{aligned}
& W_{m}=3 \int_{0}^{1} \varrho^{2 m+1} \sqrt{1-\varrho^{2}} \mathrm{~d} \varrho, \\
& \Theta_{k, \ell}=\frac{1}{2 \pi} \int_{0}^{2 \pi} \cos ^{2 k} \theta \sin ^{2 \ell} \theta \mathrm{d} \theta .
\end{aligned}
$$

Here, $m=0,1,2, \ldots$ denotes the order of the moment, and generally there are $m+1$ moments at each order $(k+\ell=m)$. For the first few orders, we find $W_{0}=1, W_{1}=2 / 5$, $W_{2}=8 / 35$, and in general $W_{m}=2 m /(2 m+3) W_{m-1}$. Similarly, for the angular integrals, $\Theta_{0,0}=1, \Theta_{1,0}=\Theta_{0,1}=1 / 2, \Theta_{2,0}=\Theta_{0,2}=3 / 8, \Theta_{1,1}=1 / 8$, etc. Using

$$
\Theta_{m, 0}=\frac{1 \times 3 \times \ldots \times(2 m-1)}{2 \times 4 \times \ldots \times(2 m)}
$$




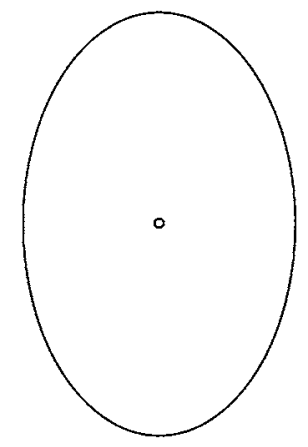

Figure 1. The order-0 model.
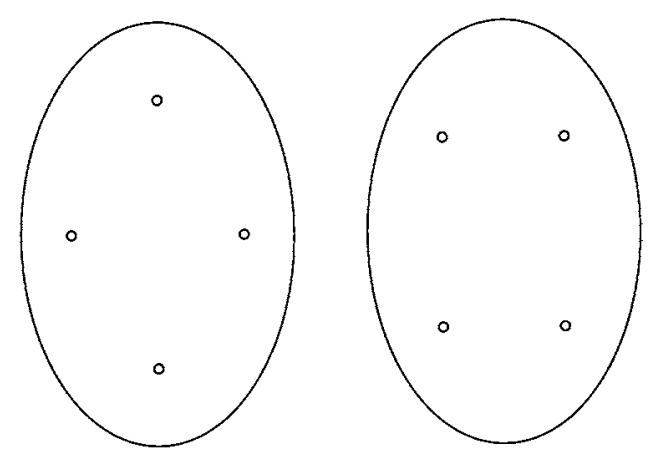

FIGURE 2. The order-1 model.

for $m>0$, together with $\Theta_{k, \ell}=\Theta_{\ell, k}$ and $\Theta_{k+1, \ell}+\Theta_{k, \ell+1}=\Theta_{k, \ell}$, we can find any value of $\Theta_{k, \ell}$ recursively.

The corresponding moments for a set of point vortices confined to the middle-major axis plane are

$$
\mu_{k, \ell}=\sum_{j=1}^{n} \kappa_{j} \tilde{y}_{j}^{2 k} \tilde{z}_{j}^{2 \ell},
$$

where $\tilde{y}_{j}=\hat{\boldsymbol{b}} \cdot\left(\boldsymbol{x}_{j}-\boldsymbol{X}\right)$ and $\tilde{z}_{j}=\hat{\boldsymbol{c}} \cdot\left(\boldsymbol{x}_{j}-\boldsymbol{X}\right)$. There are also moments involving odd powers of $\tilde{y}_{j}$ and $\tilde{z}_{j}$, and to ensure that these all vanish, we must choose the points $\tilde{y}_{j}$ and $\tilde{z}_{j}$ symmetrically, i.e. if $\tilde{y}_{j} \neq 0$, there must be another point with $\tilde{y}_{i}=-\tilde{y}_{j}$ having $\kappa_{i}=\kappa_{j}$, and likewise for $\tilde{z}_{j} \neq 0$. This means that we can either have a single point at the origin $\tilde{y}_{j}=\tilde{z}_{j}=0$, a pair lying on either the $\tilde{y}$ or the $\tilde{z}$ axis, or a quartet with both $\tilde{y}_{j}$ and $\tilde{z}_{j}$ non-zero. Here, for reasons of computational efficiency, we want to determine the minimum number of point vortices $n$ needed to match all moments of the exact elliptic sheet (3.6) up to a given order $m$. To do this requires the simultaneous solution of a set of algebraic equations ( $m$ th degree polynomials in $\tilde{y}_{j}^{2}$ and $\tilde{z}_{j}^{2}$ ) of growing complexity, and we have not been able to find the general solution for arbitrary $m$. Fortunately, however, the solutions for just $m=1$ or 2 are sufficiently accurate in practice that nothing more is required (see $\S 5$ below).

We summarize next the solutions for orders $m=0$ to 3 .

$m=0$. Here only one point vortex is needed (figure 1):

- $\kappa_{1}=\kappa$ at $\varrho=0$.

$m=1$. Here, $n=4$ point vortices are needed (figure 2): 

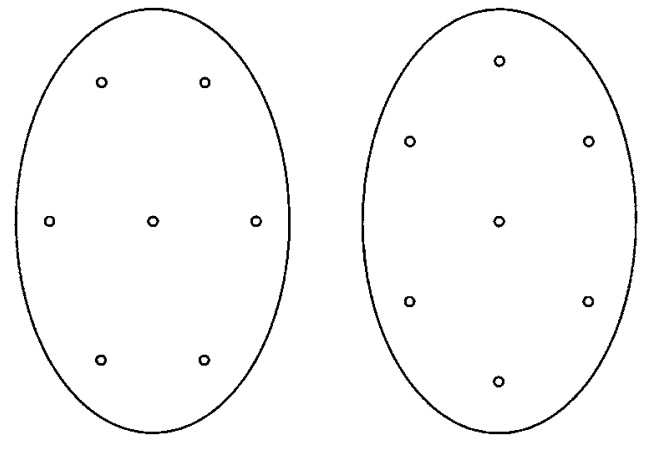

FIGURE 3. The order-2 model.
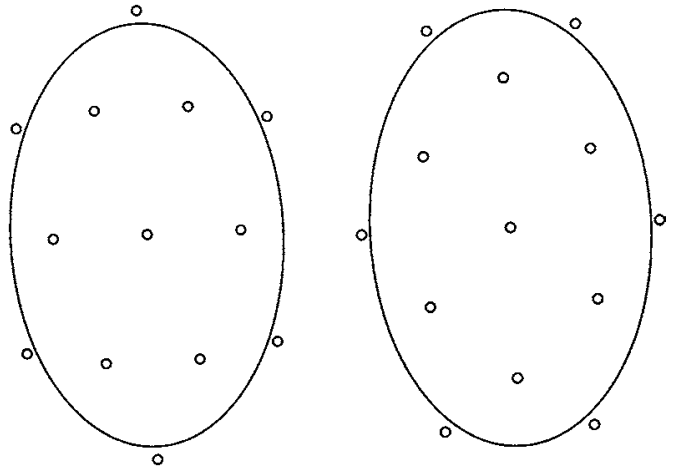

FIGURE 4. The order-3 model.

- a quartet with $\kappa_{j}=\kappa / 4$ on the ring $\varrho=\sqrt{2 / 5}$, i.e.

$$
\tilde{y}_{j}=\eta \varrho \cos \theta_{j}, \quad \tilde{z}_{j}=\tau \varrho \sin \theta_{j},
$$

with either $\theta_{j}=j \pi / 2$ or $j \pi / 2-\pi / 4$.

The configuration on the right proves to be more accurate in terms of its representation of $\psi$ exterior to the ellipsoid (as measured by comparing with the exact solution in Chandrasekhar 1969).

$m=2$. Here, $n=7$ point vortices are needed (figure 3):

- a sextet with $\kappa_{j}=7 \kappa / 60$ equally spaced in $\theta_{j}$ on the ring $\varrho=\sqrt{4 / 7}$, with either $\theta_{j}=j \pi / 3$ or $j \pi / 3-\pi / 6$, plus

- a point vortex at the origin $\varrho=0$ with $\kappa_{7}=3 \kappa / 10$.

The configuration on the right proves to be more accurate in practice.

$m=3$. Here, $n=13$ point vortices are needed (figure 4$)$ :

- an outer sextet with $\kappa_{j}=(18-19 \sqrt{2 / 3}) \kappa / 280 \simeq 0.008880589 \kappa$ equally spaced in $\theta_{j}$ on the ring $\varrho=[(4+2 \sqrt{2 / 3}) / 5]^{1 / 2} \simeq 1.06141351$, plus

- an inner sextet with $\kappa_{j}=(18+19 \sqrt{2 / 3}) \kappa / 280 \simeq 0.119690839 \kappa$ equally spaced in $\theta_{j}$, but shifted by $\pi / 6$ with respect to the outer sextet, on the $\operatorname{ring} \varrho=[(4-2 \sqrt{2 / 3}) / 5]^{1 / 2} \simeq$ 0.68804169 , plus

- a point vortex at the origin $\varrho=0$ with $\kappa_{13}=8 \kappa / 35 \simeq 0.228571429 \kappa$.

Again, the configuration on the right proves to be more accurate in practice. 
Note that the residual error in $\psi$ is $O\left(r^{-(2 m+3)}\right)$ when moments are matched to order $m$. This has been verified by comparing the discrete approximation to $\psi(3.1)$ with the exact result involving elliptic integrals (Laplace 1784) given by (B 1) in Appendix B.

In the ellipsoidal model, we need to integrate $\psi\left(\boldsymbol{x}^{\prime}\right)$ over the volume of another ellipsoid to determine the interaction energy $H_{i}$ - see $(2.24 c)$. As in the two-dimensional elliptical model, we use the same quadrature formula to carry out this integration (even though all the quadrature points lie in the middle-major axis plane), since this preserves the symmetry of $H_{i}$ (and therefore preserves all conservation laws of the original system). The resulting discrete form of $H_{i}$ used in the model is

$$
H_{i}=\sum_{i=1}^{n} \sum_{j=1}^{n} \frac{\kappa_{i}^{\prime} \kappa_{j}}{\left|\boldsymbol{x}_{i}^{\prime}-\boldsymbol{x}_{j}\right|},
$$

where $\boldsymbol{x}_{i}^{\prime}=\boldsymbol{X}^{\prime}+\tilde{y}_{i}^{\prime} \hat{\boldsymbol{b}}^{\prime}+\tilde{z}_{i}^{\prime} \hat{\boldsymbol{c}}^{\prime}$ and $\boldsymbol{x}_{j}=\boldsymbol{X}+\tilde{y}_{j} \hat{\boldsymbol{b}}+\tilde{z}_{j} \hat{\boldsymbol{c}}$. This expression is identical to the Hamiltonian for a system of independent point vortices. In the ellipsoidal model, however, the point vortices are not independent, since they are constrained to move with each ellipsoid.

In Appendix C, we show that the 'ellipsoidal moment model' of Miyazaki et al. (2001) may be derived by expanding $H_{i}$ about the centroid separation $\boldsymbol{R}=\boldsymbol{X}^{\prime}-\boldsymbol{X}$, i.e. using $\boldsymbol{x}_{i}^{\prime}-\boldsymbol{x}_{j}=\boldsymbol{R}+\boldsymbol{r}_{i j}$, where $\boldsymbol{r}_{i j}$ is the remainder, presumed small compared to $\boldsymbol{R}$, then expanding $H_{i}$ to second order in $\boldsymbol{r}_{i j} /|\boldsymbol{R}|$. The resulting expression is independent of the number of point vortices $n$ and therefore applies as $n \rightarrow \infty$.

\section{The numerical algorithm}

A brief sketch of the numerical algorithm developed for the ellipsoidal model is provided next.

Initialization. The order $m$ is selected and the constant values of $\kappa_{j} / \kappa, \tilde{y}_{j} / \eta$ and $\tilde{z}_{j} / \tau$ are stored. These are independent of the vortex strength $\kappa$, shape $\mathscr{B}$ and position $\boldsymbol{X}$. The number of ellipsoids is read in, and then $q, \mathscr{B}$ and $\boldsymbol{X}$ are read in for each ellipsoid. The time step $\Delta t$ is selected (see below).

(i) Self-induced motion. At the beginning of each time step, a $3 \times 3$ eigenvalue problem is solved for each ellipsoid to determine the eigenvalues $a^{2}, b^{2}$ and $c^{2}$, and the associated (unit) eigenvectors $\hat{\boldsymbol{a}}, \hat{\boldsymbol{b}}$ and $\hat{\boldsymbol{c}}$ from the shape matrix $\mathscr{B}$. These eigenvectors make up the rotation matrix $\mathscr{M}$, see (2.2). From $a^{2}, b^{2}$ and $c^{2}$, the elliptic integrals in (2.19) are computed to give $\mathscr{P}_{v}=\mathscr{M} \mathscr{D} \mathscr{M}^{T}$ and hence the self-induced flow matrix $\mathscr{S}_{v}=\mathscr{L} \mathscr{P}_{v}$ needed for the evolution of $\mathscr{B}$, cf. (2.4). There is no self-induced contribution to $\boldsymbol{X}_{t}$. Some preparatory work is done for the following step, such as computing the point vortex locations $\boldsymbol{x}_{j}$ within each vortex.

(ii) Interaction between separate vortices. Next, all pairs of distinct vortices, $\{q, \boldsymbol{X}, \mathscr{B}\}$ and $\left\{q^{\prime}, \boldsymbol{X}^{\prime}, \mathscr{B}^{\prime}\right\}$, are considered. The derivatives of $H_{i}$ with respect to $\boldsymbol{X}$ and $\mathscr{B}$ are calculated explicitly (see Appendix D) to give $\boldsymbol{U}_{b}$ and $\mathscr{S}_{b}$ from (2.16).

(iii) Time integration. The above contributions (i) and (ii) to $\boldsymbol{U}$ and $\mathscr{S}$ are summed together, then the right-hand side of $(2.4 b)$ is computed for each vortex separately, and the values of $\boldsymbol{X}$ and $\mathscr{B}$ are updated using a fourth-order Runge-Kutta numerical integrator.

The time step $\Delta t$ is chosen to be less than or equal to $\pi /\left(10|q|_{\max }\right)$, where $|q|_{\max }$ is the maximum PV magnitude. Smaller time steps are used for longer integrations, to keep the accumulated error ( $\propto T_{\operatorname{sim}} \Delta t^{4}$, where $T_{\text {sim }}$ is the duration of the simulation) small. 


\section{Numerical results}

We have performed a series of tests to check the consistency and accuracy (with respect to the full QG equations) of the numerical algorithm. We have verified that the energy, or $H$, is conserved within errors expected from the time-stepping scheme (the error decreases like $\Delta t^{4}$ ), for long integrations starting with various unsteady asymmetric configurations of two or more vortices. The model also conserves the angular momentum $J_{\text {tot }}$, or impulse, to which each vortex contributes

$$
\begin{aligned}
J & =\iiint_{V} q\left(x^{2}+y^{2}\right) \mathrm{d} V, \\
& =4 \pi \kappa\left(X^{2}+Y^{2}+\frac{1}{5}\left(\mathscr{B}_{11}+\mathscr{B}_{22}\right)\right) .
\end{aligned}
$$

This is also conserved to within time-stepping errors. The model exactly conserves the linear momentum $\boldsymbol{I}_{\text {tot }}$, or impulse, to which each vortex contributes

$$
\begin{aligned}
\boldsymbol{I} & =\iiint_{V} q \boldsymbol{x} \mathrm{d} V, \\
\boldsymbol{I} & =4 \pi \kappa \boldsymbol{X} .
\end{aligned}
$$

This follows from the symmetric form of $H_{i}$ in (3.10) and (D 1). Finally, the model conserves the volume (proportional to $a b c=|\mathscr{B}|^{1 / 2}$ ) of each vortex, again to within time-stepping errors.

The next test compares the predictions of the ellipsoidal model and of the full QG equations for the initial tendencies $\boldsymbol{X}_{t}$ and $\mathscr{B}_{t}$ in an example of two closely interacting ellipsoids. This is a non-trivial comparison since, in the full QG equations, the flow does not exactly preserve ellipsoidal shapes. Here, we are measuring how well the ellipsoidal model captures the ellipsoidal deformations induced by the exact (QG) dynamics.

The base configuration consists of nearly touching vortices, each having moderate deformation and more-or-less arbitrary orientation. Each vortex has the same PV, $q=q^{\prime}=4 \pi$, while the centres are chosen as $\boldsymbol{X}=(-0.7,-0.5,-0.3)$ and $\boldsymbol{X}^{\prime}=(0.6$, $0.6,0.3)$. The shape matrices $\mathscr{B}$ and $\mathscr{B}^{\prime}$ are generated from specified axes lengths $(a, b, c)$ and Euler angles $(\alpha, \beta, \gamma)$ as follows:

$$
\begin{aligned}
& \hat{\boldsymbol{a}}=(\cos \beta \cos \alpha \cos \gamma-\sin \alpha \sin \gamma, \cos \beta \sin \alpha \cos \gamma+\cos \alpha \sin \gamma,-\sin \beta \cos \gamma), \\
& \hat{\boldsymbol{b}}=(-\cos \beta \cos \alpha \sin \gamma-\sin \alpha \cos \gamma,-\cos \beta \sin \alpha \sin \gamma+\cos \alpha \cos \gamma, \sin \beta \sin \gamma), \\
& \hat{\boldsymbol{c}}=(\sin \beta \cos \alpha, \sin \beta \sin \alpha, \cos \beta), \\
& \mathscr{B}=a^{2} \hat{\boldsymbol{a}} \hat{\boldsymbol{a}}^{T}+b^{2} \hat{\boldsymbol{b}} \hat{\boldsymbol{b}}^{T}+c^{2} \hat{\boldsymbol{c}} \hat{\boldsymbol{c}}^{T}
\end{aligned}
$$

and similarly for $\mathscr{B}^{\prime}$. Here we take $(a, b, c)=(0.6,0.8,1.0),(\alpha, \beta, \gamma)=\left(20^{\circ}, 40^{\circ}, 60^{\circ}\right)$, $\left(a^{\prime}, b^{\prime}, c^{\prime}\right)=(0.5,0.8,1.1)$, and $\left(\alpha^{\prime}, \beta^{\prime}, \gamma^{\prime}\right)=\left(30^{\circ}, 50^{\circ}, 70^{\circ}\right)$. The resulting configuration is shown in figure 5 from two perspectives to emphasize just how close the two vortices are.

To compare with the full QG dynamics, we use the contour surgery algorithm (referred to as contour dynamics hereinafter), described in the appendix to Dritschel (2002). In this algorithm, the fluid is modelled by a fixed number of layers $n_{\text {lay }}$ within which the PV contours bounding the vortices move horizontally. All fields, like the PV and the velocity field $\boldsymbol{u}$, are vertically averaged over each layer. This averaging results in an error of $O\left(n_{\text {lay }}^{-2}\right)$. In addition, there are errors associated with the finite point spacing along the PV contours in each layer. We have ensured that the maximum 

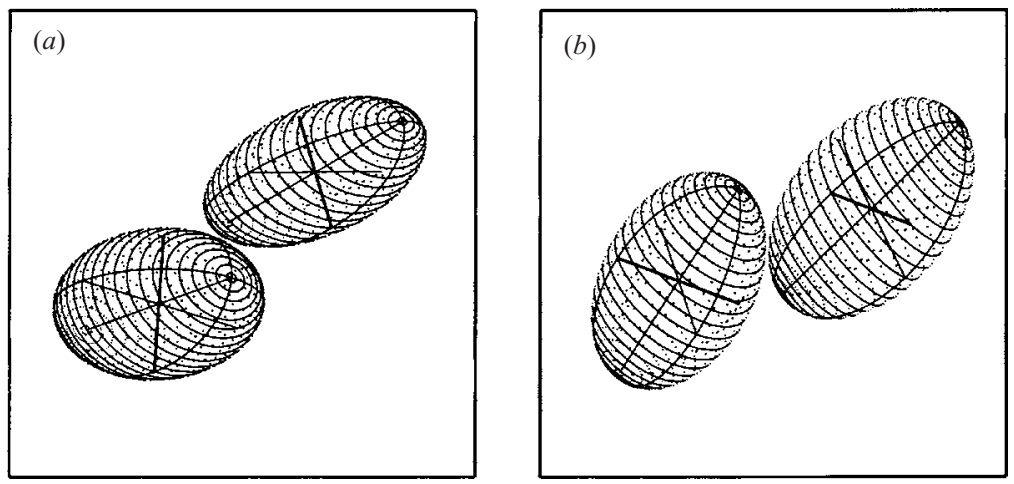

FIgURE 5. $(a)$ Top and $(b)$ side views of the base configuration. The side view is from a longitude of $-60^{\circ}$ (between the positive $x$ and negative $y$ axes) in the equatorial plane $z=0$. The minor axis is rendered as a bold line, while the middle and major axes are rendered as thin lines. Ellipses are drawn on the surface of each ellipsoid in planes perpendicular to the major axis, and dashed lines are used for the far sides of each ellipsoid.

point spacing $\mu L$ is no larger than the layer thickness, where $\mu$ is a dimensionless parameter and $L$ is the largest axis length in either ellipsoid at the initial time.

In normal applications, a small to moderate number of layers (20-40) is usually sufficient to obtain reasonably accurate simulation results. Here, however, we have had to use a much larger number of layers, $n_{\text {lay }}=1000$, owing to the unexpectedly high accuracy of the ellipsoidal model. Correspondingly, we have taken $\mu=0.006$, giving a total number of contour points of nearly 770000 . Just to compute the velocity field once took nearly 5 days of computer time on a $500 \mathrm{MHz}$ alpha processor (the cost of contour dynamics is proportional to the square of the number of points).

This velocity field, specifically $\boldsymbol{u}$ at all contour points $\boldsymbol{x}=\overline{\boldsymbol{x}}$ lying on the surface of the ellipsoid $\{q, \boldsymbol{X}, \mathscr{B}\}$, is used to calculate $\boldsymbol{X}_{t}$ and $\mathscr{B}_{t}$ at the initial instant of time. This is done using the contour integral representations of $\boldsymbol{X}$ and $\mathscr{B}$, namely

$$
\begin{aligned}
& \boldsymbol{X}=\frac{2 \Delta z}{3 V} \sum_{\ell=1}^{n_{\text {lay }}} \oint \overline{\boldsymbol{x}}_{\ell}\left(\bar{x}_{\ell} \mathrm{d} \bar{y}_{\ell}-\bar{y}_{\ell} \mathrm{d} \bar{x}_{\ell}\right), \\
& \mathscr{B}=\frac{5 \Delta z}{4 V} \sum_{\ell=1}^{n_{\text {lay }}} \oint\left(\overline{\boldsymbol{x}}_{\ell}-\boldsymbol{X}\right)\left(\overline{\boldsymbol{x}}_{\ell}-\boldsymbol{X}\right)^{T}\left(\bar{x}_{\ell} \mathrm{d} \bar{y}_{\ell}-\bar{y}_{\ell} \mathrm{d} \bar{x}_{\ell}\right),
\end{aligned}
$$

where $\Delta z$ is the layer thickness, $\overline{\boldsymbol{x}}_{\ell}$ is a point on the contour in layer $\ell$ and $V$ is the volume of the vortex, computed from

$$
V=\frac{1}{2} \Delta z \sum_{\ell=1}^{n_{\text {lay }}} \oint\left(\bar{x}_{\ell} \mathrm{d} \bar{y}_{\ell}-\bar{y}_{\ell} \mathrm{d} \bar{x}_{\ell}\right) .
$$

The tendencies are found by taking a time derivative of (5.4) and substituting $\mathrm{d} \overline{\boldsymbol{x}}_{\ell} / \mathrm{d} t=\boldsymbol{u}\left(\overline{\boldsymbol{x}}_{\ell}\right)$, the velocity at each contour point. The contour integrals are computed by two-point Gaussian quadrature along cubic splines connecting the original set of contour points; we have taken care to evaluate $\boldsymbol{u}$ directly at these quadrature points to avoid additional interpolation errors.

The initial contours are found by first shifting and dilating the configuration so that it lies in the range $0 \leqslant z \leqslant 1$. Contours are then created by intersecting the mean height of each layer with the ellipsoids. From these contours, we recalculate $\boldsymbol{X}, \boldsymbol{X}^{\prime}, \mathscr{B}$ and 

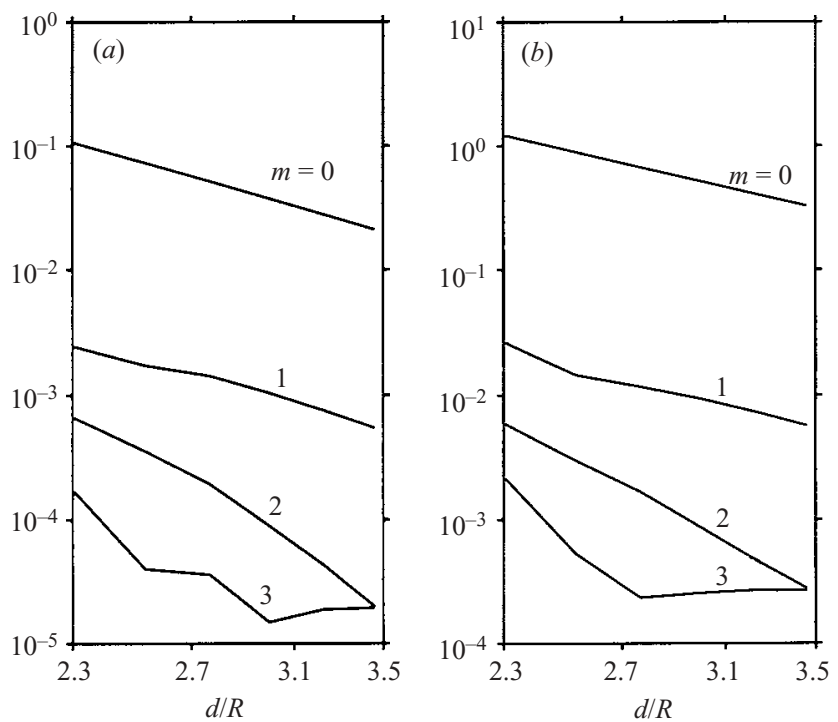

Figure 6. Differences between the ellipsoidal model at various orders $m$ and the full QG dynamics (using contour dynamics with 1000 layers): $(a)$ the centroid tendency error $\left|\Delta \boldsymbol{X}_{t}\right|$, and $(b)$ the shape matrix tendency error $\left|\Delta \mathscr{B}_{t}\right|$, versus the dimensionless vortex separation $d / R$.

$\mathscr{B}^{\prime}$ using (5.4) for use in the ellipsoidal model to give the closest possible comparison. We also calculate $\boldsymbol{X}_{t}$ and $\mathscr{B}_{t}$ in both contour dynamics and the ellipsoidal model. Error is measured by the r.m.s. differences (or $L_{2}$ norms) $\left|\Delta \boldsymbol{X}_{t}\right|$ and $\left|\Delta \mathscr{B}_{t}\right|$.

Several other configurations differing only in the initial centroid separation $\boldsymbol{X}-\boldsymbol{X}^{\prime}$ have also been examined to determine how $\left|\Delta \boldsymbol{X}_{t}\right|$ and $\left|\Delta \mathscr{B}_{t}\right|$ depend on the distance between the vortex centres $d=\left|\boldsymbol{X}-\boldsymbol{X}^{\prime}\right|$. These other configurations are obtained by multiplying $\boldsymbol{X}$ and $\boldsymbol{X}^{\prime}$ by a sequence of magnification factors: $1.1,1.2, \ldots, 1.5$. The results are summarized in figure 6 , which plots $\left|\Delta \boldsymbol{X}_{t}\right|$ and $\left|\Delta \mathscr{B}_{t}\right|$ versus $d / R$ (in $\log -\log$ scaling), where $R=(a b c)^{1 / 3}$ is the mean radius of the larger vortex, for the ellipsoidal model at orders $m=0,1,2$ and 3 (using, respectively, $n=1,4,7$ and 13 point vortices per vortex to represent the interaction). Note that in these plots, the errors have been scaled to correspond to the original configurations (before fitting them into $0 \leqslant z \leqslant 1$ ); in particular, the vortex volumes are independent of $d / R$. For a given order $m,\left|\Delta \mathscr{B}_{t}\right| \sim 10\left|\Delta \boldsymbol{X}_{t}\right|$, indicating that the shape evolution is less reliable than the centroid evolution. This is sensible since $\boldsymbol{X}$ corresponds to a lower-order spatial moment, $V^{-1} \iiint_{V} \boldsymbol{x} \mathrm{d} V$, than does $\mathscr{B}$, see (2.14). However, half of this difference may be due to the definition of $\mathscr{B}$ as 5 times the second-order spatial moment. Both $\left|\Delta \boldsymbol{X}_{t}\right|$ and $\left|\Delta \mathscr{B}_{t}\right|$ decrease in all cases as fast or faster than $(R / d)^{2 m+4}$ (not shown), which is the error expected from the expansion (3.2) for large $d / R$ (with $r \sim d$ ). Also, the errors for $m>0$ are remarkably small, even for nearly touching vortices. The error is only $\sim 10^{-3}$ for $m=2$. This level of error is comparable with or less than the error associated with contour dynamics using a normal number of layers. When $m=3$, the error in the ellipsoidal model is so small that not even 1000 layers is sufficiently accurate in contour dynamics to measure it - note the saturation of error for $d / R \gtrsim 2.8$.

The exceptional case is $m=2$ : here the error decreases nearly as fast as for $m=3$, and perhaps this is because the point vortex configuration for $m=2$ is not very 
different from that for $m=3$, compare figures 3 and 4 , and recall that the outer sextet of vortices in figure 4 have only $7 \%$ of the strength of the inner sextet. Indeed, if we compare the circulations of the inner sextet for $m=3$ with those of the sextet for $m=2$, we find that they differ by only $2.5 \%$ while the ring radius $\varrho$ differs by $9 \%$. Moreover, the $m=2$ configuration comes within $18.6 \%$ (in r.m.s. terms) of the numerical constants $W_{k+\ell} \Theta_{k, \ell}$ multiplying sixth-order moment coefficients $\mu_{k, \ell}$, with $k+\ell=3$, in (3.6). It is fortuitous that just 7 point vortices can model the dynamics of interacting ellipsoids so well.

We next examine the differences between the ellipsoidal model and contour dynamics in the evolution of the flow. These same six configurations were integrated to $t=20$ (note that the period of rotation of an isolated spherical volume having $q=4 \pi$ is $6 \pi / q=1.5$ ). In the contour dynamics simulations, the vortices develop non-ellipsoidal disturbances, and we are interested in finding out how much these disturbances matter in terms of the prediction of the vortex centres and mean ellipsoidal shapes. Note, for the configurations having a magnification less than or equal to $1.3(d / R \lesssim 3.0)$, the vortices actually merge in contour dynamics, making it difficult to compare with the ellipsoidal model thereafter. The case 1.3 exhibits only momentary merging, and we are able to compare before and after the period when the vortices are joined together.

In all of the contour dynamics simulations, we used 50 layers and $\mu=0.12$, because of computational limitations. A greater number of layers would improve the comparisons shown below, but they are in any case indicative. The first comparison focuses on a case without any merging, magnification $1.4(d / R \approx 3.228)$. Four ellipsoidal simulations, differing only in the order $m$, are compared with the contour dynamics simulation, shown in figure 7 together with the ellipsoidal model simulation for $m=2$. Note that the contour dynamics simulation required $4785 \mathrm{~s}$ on a $500 \mathrm{MHz}$ alpha processor, while the ellipsoidal model (for $m=0,1,2$ and 3 ) required just 0.588, $0.633,0.688$ and $0.919 \mathrm{~s}$. The two simulations agree remarkably well, despite clear non-ellipsoidal deformations, even filamentation, in the contour dynamics simulation. We quantify this by measuring the joint r.m.s. error in $\boldsymbol{X}$ and $\boldsymbol{X}^{\prime}$, defined by

$$
|\Delta \boldsymbol{X}|_{m}^{2}=\frac{1}{2}\left(\left|\boldsymbol{X}_{m}-\boldsymbol{X}_{\mathrm{CD}}\right|^{2}+\left|\boldsymbol{X}_{m}^{\prime}-\boldsymbol{X}_{\mathrm{CD}}^{\prime}\right|^{2}\right),
$$

where a subscript $m$ refers to the ellipsoidal model at order $m$ and a subscript CD refers to contour dynamics. The joint r.m.s. error in $\mathscr{B}$ and $\mathscr{B}^{\prime}$ is defined similarly (in terms of differences in the components). The time evolution of the differences $|\Delta \boldsymbol{X}|_{m}$ and $|\Delta \mathscr{B}|_{m}$ is shown in figure 8 . Note that in the same norm $|\mathscr{B}| \approx 1.318$ at $t=0$, so the late-time errors $|\Delta \mathscr{B}|_{m}$ for $m=2$ and $m=3$ are approximately $15 \%$. The centroid errors are considerably smaller, only about $2.0 \%$ of the initial centroid separation between the vortices and about $6.4 \%$ of the radius of the largest vortex. (Part of this may be due to the factor of 5 in (2.14) as discussed above.) Order $m=0$ clearly stands out - this model is too crude to capture the ellipsoidal deformations which contribute significantly to the dynamics, as can be seen by the close comparison for $m>0$. With increasing order, the differences diminish, but the errors in the contour dynamics simulation are too great to distinguish $m=2$ and $m=3$. Another interpretation is that the remaining differences, mostly due to non-ellipsoidal deformations (see below), cannot be significantly reduced by increasing the order of the ellipsoidal model beyond $m=2$. Yet, clearly there is an advantage of using $m=2$ over $m=1$.

In a final comparison, we fix the order of the ellipsoidal model at $m=2$ and examine how the ellipsoidal model compares with contour dynamics for the 6 magnifications, 1.0-1.5. Figure 9 plots the time evolution of the differences $|\Delta \boldsymbol{X}|_{m}$ and $|\Delta \mathscr{B}|_{m}$, over time intervals when the vortices are separated (this is only a short time for 
(a)

(b)

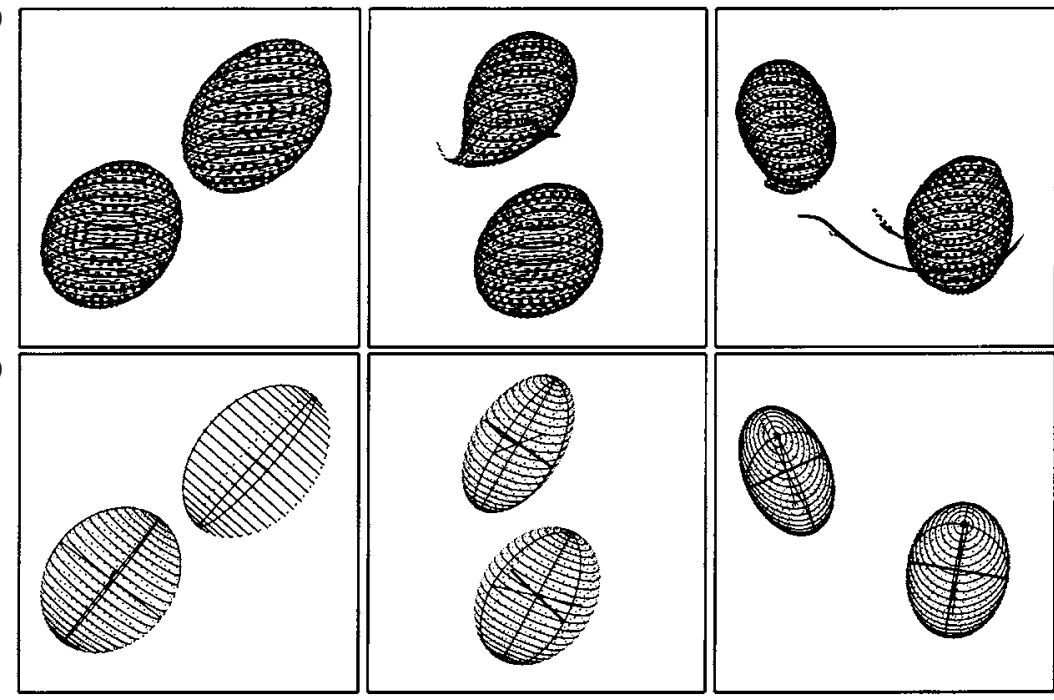

$(c)$

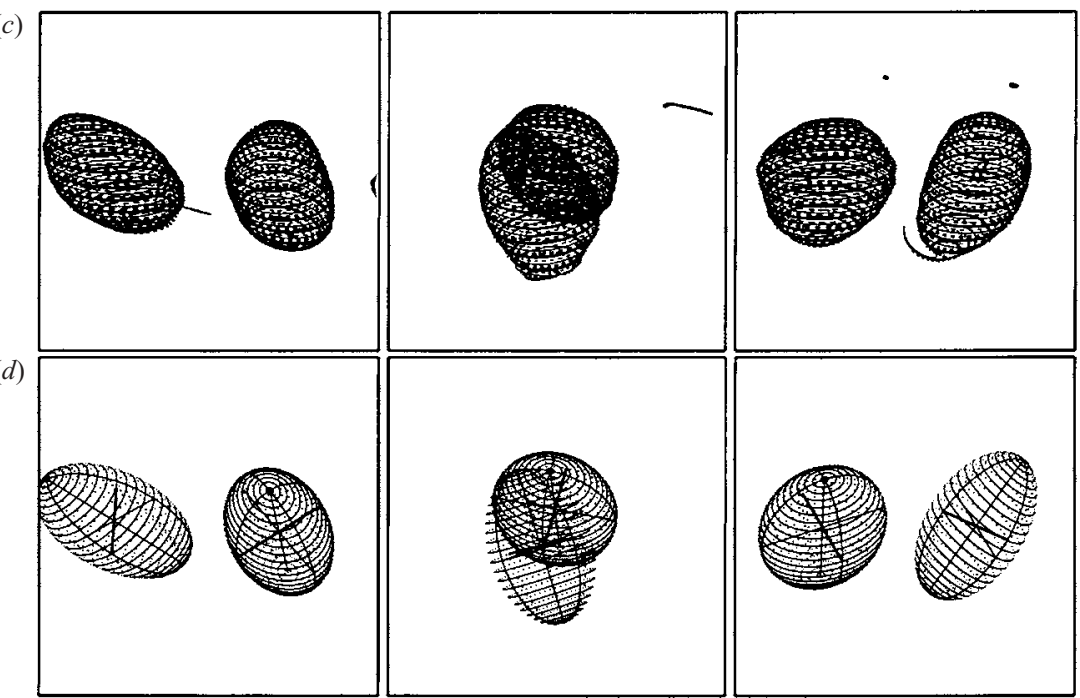

Figure 7. Comparison of the QG contour dynamics simulation $(a, c)$ and the ellipsoidal model simulation for $m=2(b, d)$ at times $t=0,4,8(a, b)$ and $t=12,16$ and $20(c, d)$, for the magnification 1.4 case. The vortices are viewed orthographically, at an angle of $60^{\circ}$ from the vertical, and in the plane $y=0$. The contours in the QG simulation lie in horizontal planes (here 50 layers are used altogether). In the ellipsoidal model simulation, the ellipsoids are rendered as described in figure 5.

magnifications 1.0-1.2). The differences rapidly diminish as expected with increasing magnification, or $d / R$, even though the vortices in the contour dynamics simulations exhibit strong non-ellipsoidal deformations, even for magnification $1.5(d / R \approx 3.458)$. The ellipsoidal model appears to be a remarkably good predictor of the ellipsoidal component of the dynamics for non-merging vortices.

The non-ellipsoidal deformations evidently feed back only very weakly on the ellipsoidal component of the dynamics. To check this feedback, we have compared the ellipsoidal model with contour dynamics for all six magnifications at early times, before merger. During this time, non-ellipsoidal disturbances are growing, and while 

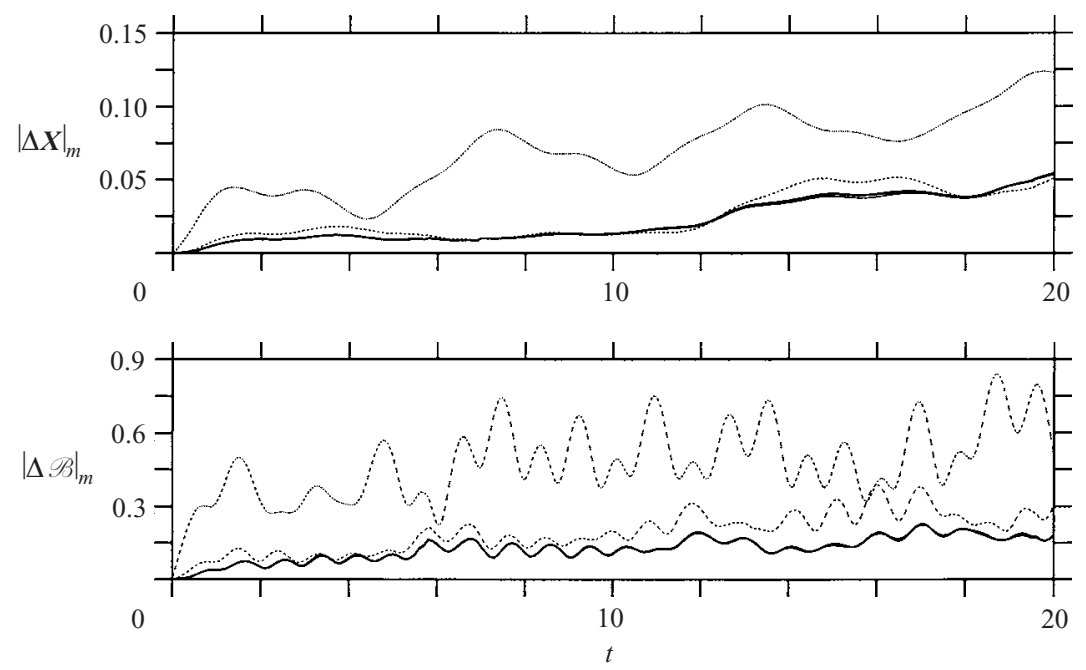

Figure 8. Time evolution of the differences between the ellipsoidal model at various orders $m$ and the full QG dynamics (computed by contour dynamics with $n_{\text {lay }}=50$, see figure 7). The line styles are as follows: $m=0$ short-dashed, $m=1$ long-dashed, $m=2$ thin-solid, $m=3$ bold-solid.
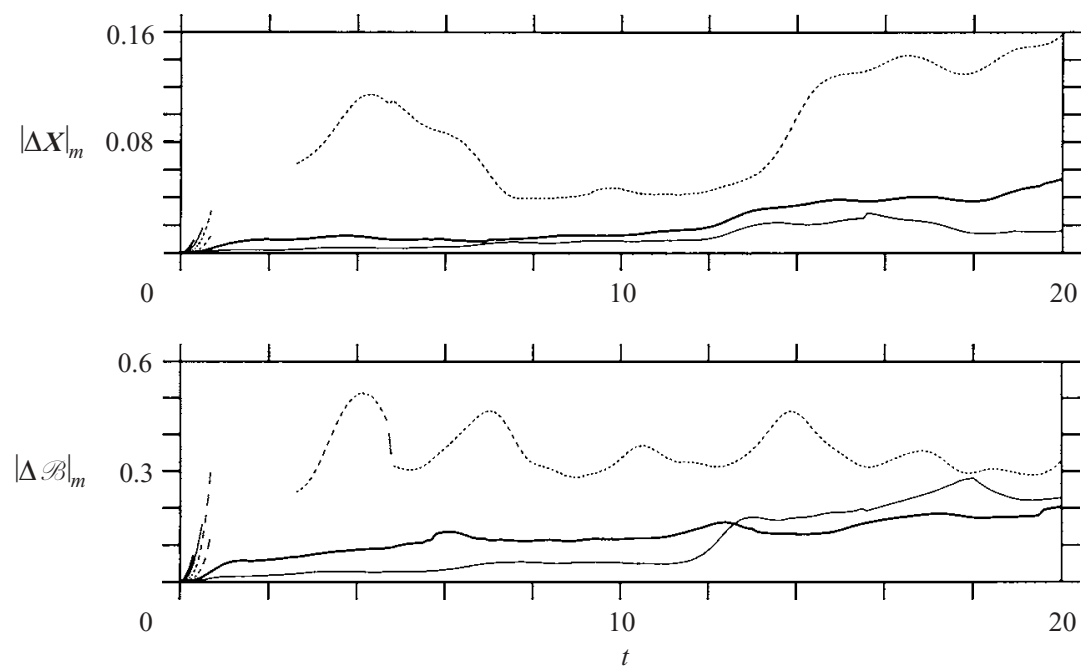

FIGURE 9. Time evolution of the differences between the ellipsoidal model at order $m=2$ and the full QG dynamics for various magnifications: 1.0 bold, 1.1 thin, 1.2 short-dashed, 1.3 long-dashed, 1.4 bold, and 1.5 thin. Note that the curves for the first three magnifications exist only near $t=0$, while the fourth has a break between $t=0.8$ and 2.5 when the two initial vortices are joined together in a dumbbell shape.

they are not yet at peak amplitude, their existence is sufficient to estimate the way in which the feedback depends on the distance $d$ between vortex centres. Let $d_{0}$ denote the distance between vortex centres in the original, unmagnified case $\left(d_{0} \approx 2.306 R\right)$. Then $d / d_{0}$ gives the magnification. The feedback is quantified by computing the r.m.s. values of $|\Delta \boldsymbol{X}|_{m}$ and $|\Delta \mathscr{B}|_{m}$ over $0 \leqslant t \leqslant 0.4$ and for orders $m=2$ and 3 . The original 50 -layer contour dynamics simulations proved too inaccurate for this comparison (the $|\Delta \boldsymbol{X}|$ error saturates for $d / d_{0}=1.4$ and 1.5 ), so the resolution was increased 


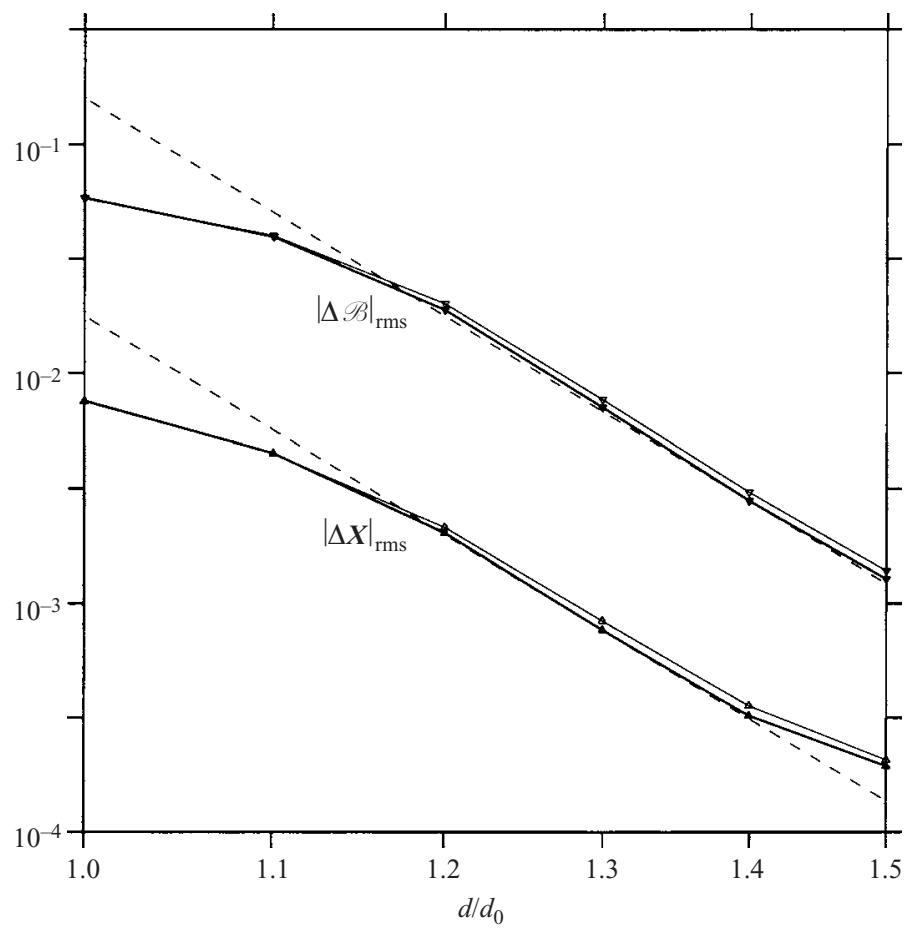

FIGURE 10. The dependence of the early-time r.m.s. differences $|\Delta \boldsymbol{X}|_{\mathrm{rms}}$ and $|\Delta \mathscr{B}|_{\mathrm{rms}}$ between the full QG dynamics and the ellipsoidal model at order $m=2$ (thin solid lines) and at order $m=3$ (bold solid lines). Two slopes proportional to $\left(d / d_{0}\right)^{-12}$ are also indicated by the dashed lines.

four-fold to 200 layers (and $\mu=0.03$ ). These higher-resolution simulations were run only until $t=0.4$, requiring about 5 hours of computer time per run. The results are shown in figure 10 , in log-log scaling, together with two lines corresponding to a constant $\times\left(d / d_{0}\right)^{-12}$. This dependence, on the minus twelfth power of the distance between vortex centres, is surprisingly steep. (The saturation of $|\Delta \boldsymbol{X}|_{\mathrm{rms}}$ for larger $d / d_{0}$ is believed to be due to an insufficient number of layers in the contour dynamics simulation.) There is little difference between $m=2$ and $m=3$, so what we see here is truly a measure of the effect of non-ellipsoidal deformations on the ellipsoidal component of the dynamics. This dependence decays very rapidly indeed with vortex separation.

At later times, the dependence on $d / d_{0}$ may become much shallower, but for the present examples it is difficult to quantify since the flow evolution is strongly dependent on $d / d_{0}$, with merging at small distances.

\section{Concluding remarks}

In this paper, we have derived a simplified model for vortex interactions in rotating stratified flows, in the quasi-geostrophic regime. The single assumption in our model is that each vortex has an ellipsoidal shape, tantamount to replacing the infinite degrees of freedom of the original system by just 9 degrees of freedom per vortex. We then showed how the Hamiltonian structure of the full equations can be carried over to the reduced, ellipsoidal model, and that this structure is sufficient to generate the dynamical evolution equations for the model. 
The exact Hamiltonian, however, cannot be evaluated efficiently, and instead a discrete 'point-vortex' approximation was introduced to calculate the interaction Hamiltonian. In numerical tests, this was shown to be very accurate for as few as 7 point vortices per ellipsoid. In fact, there appears to be no reason to use more point vortices, since non-ellipsoidal deformations (neglected in the model) prove to be more important than the accuracy of the point-vortex approximation. However, 7 point vortices capture the fourth and much of the sixth-order spatial moments of the ellipsoid, showing that these moments are important for the accuracy of the model. These moments are neglected in the ellipsoidal moment model derived by a Taylor-series expansion (Miyazaki et al. (2001); see also Appendix C).

The high accuracy of the ellipsoidal model found here in non-trivial vortex interactions has motivated a comprehensive study of the conditions leading to strong vortex interactions such as merger (Reinaud \& Dritschel 2003). The entire parameter space, for two ellipsoids, depends on 6 parameters: the PV ratio, the volume ratio, each height-to-width aspect ratio, the horizontal and the vertical offsets. So far we have a complete picture only for like-signed vortices having unit PV ratio. $\dagger$

The elliptical model in two-dimensions and the present ellipsoidal model appear to be members of a hierarchy of models of increasing spatial dimension. Though the details have not been worked out in full, many of the results presented here generalize readily to any dimension $N$ (using $N$-dimensional vectors and $N \times N$ matrices). We suspect also that the existence of a confocal family of ellipsoids (discussed in Appendix B) having the same exterior flow, which is true for both $N=2$ and 3 , also generalizes (which has direct consequences for the point vortex approximation). Furthermore, the exterior solution to Laplace's equation found by Laplace (1784) (stated in Appendix B) also appears to generalize (the solution reduces to the twodimensional one in the appropriate limit), apart from a numerical factor evidently related to the volume of a sphere in $N$ dimensions.

Support for this research has come from the UK Engineering and Physical Sciences Research Council (grant number GR/N11711). We wish to thank Michael Dritschel and Darren Crowdy for directing us to several useful references.

\section{Appendix A. Relations among elliptic integrals}

Let

$$
\Delta=\sqrt{(t+\alpha)(t+\beta)(t+\gamma)}
$$

where $\alpha, \beta$ and $\gamma$ are positive constants. The elliptic integral of the first kind is

$$
R_{F}(\alpha, \beta, \gamma)=\frac{1}{2} \int_{0}^{\infty} \frac{\mathrm{d} t}{\Delta} .
$$

Differentiation with respect to $\alpha, \beta$ and $\gamma$ generates the elliptic integral of the second kind

$$
\begin{aligned}
& R_{D}(\beta, \gamma, \alpha)=-6 \frac{\partial R_{F}}{\partial \alpha}=\frac{3}{2} \int_{0}^{\infty} \frac{\mathrm{d} t}{(t+\alpha) \Delta} \\
& R_{D}(\gamma, \alpha, \beta)=-6 \frac{\partial R_{F}}{\partial \beta}=\frac{3}{2} \int_{0}^{\infty} \frac{\mathrm{d} t}{(t+\beta) \Delta}
\end{aligned}
$$

$\dagger$ Some examples, including opposite-signed vortices, may be seen at www-vortex.mcs.st-and. ac.uk/ dgd/ELM. 


$$
R_{D}(\alpha, \beta, \gamma)=-6 \frac{\partial R_{F}}{\partial \gamma}=\frac{3}{2} \int_{0}^{\infty} \frac{\mathrm{d} t}{(t+\gamma) \Delta} .
$$

From the above expressions, and using $\mathrm{d}(t / \Delta)=\mathrm{d} t / \Delta-t \mathrm{~d} \Delta / \Delta^{2}$ together with

$$
\mathrm{d} \Delta=\frac{1}{2} \Delta\left(\frac{1}{t+\alpha}+\frac{1}{t+\beta}+\frac{1}{t+\gamma}\right) \mathrm{d} t,
$$

it is straightforward to show that

$$
\alpha R_{D}(\beta, \gamma, \alpha)+\beta R_{D}(\gamma, \alpha, \beta)+\gamma R_{D}(\alpha, \beta, \gamma)=3 R_{F}(\alpha, \beta, \gamma) .
$$

We next use these results to prove

$$
\mathscr{P}_{v}=-\frac{10}{\kappa} \frac{\partial H_{v}}{\partial \mathscr{B}}
$$

for $\mathscr{P}_{v}=\mathscr{M} \mathscr{D} \mathscr{M}^{T}$ with $\mathscr{D}$ the diagonal matrix

$$
\begin{aligned}
& \mathscr{D}_{11}=\kappa R_{D}\left(b^{2}, c^{2}, a^{2}\right), \\
& \mathscr{D}_{22}=\kappa R_{D}\left(c^{2}, a^{2}, b^{2}\right), \\
& \mathscr{D}_{33}=\kappa R_{D}\left(a^{2}, b^{2}, c^{2}\right),
\end{aligned}
$$

and for

$$
H_{v}=\frac{3}{5} \kappa^{2} R_{F}\left(a^{2}, b^{2}, c^{2}\right)
$$

Consider

$$
\frac{\partial R_{F}}{\partial \mathscr{B}}=\frac{\partial a^{2}}{\partial \mathscr{B}} \frac{\partial R_{F}}{\partial a^{2}}+\frac{\partial b^{2}}{\partial \mathscr{B}} \frac{\partial R_{F}}{\partial b^{2}}+\frac{\partial c^{2}}{\partial \mathscr{B}} \frac{\partial R_{F}}{\partial c^{2}} .
$$

To compute derivatives with respect to the matrix $\mathscr{B}$, we follow the procedure introduced by McKiver \& Dritschel (2003). We represent $\mathscr{B}$ by 6 independent components as

$$
\mathscr{B}=\left(\begin{array}{lll}
B^{1} & B^{2} & B^{3} \\
B^{2} & B^{4} & B^{5} \\
B^{3} & B^{5} & B^{6}
\end{array}\right)=\sum_{k=1}^{6} B^{k} \mathscr{J}^{k}
$$

where

$$
\begin{aligned}
& \mathscr{J}^{1}=\left(\begin{array}{lll}
1 & 0 & 0 \\
0 & 0 & 0 \\
0 & 0 & 0
\end{array}\right) \quad \mathscr{J}^{2}=\left(\begin{array}{lll}
0 & 1 & 0 \\
1 & 0 & 0 \\
0 & 0 & 0
\end{array}\right) \quad \mathscr{J}^{3}=\left(\begin{array}{lll}
0 & 0 & 1 \\
0 & 0 & 0 \\
1 & 0 & 0
\end{array}\right), \\
& \mathscr{J}^{4}=\left(\begin{array}{lll}
0 & 0 & 0 \\
0 & 1 & 0 \\
0 & 0 & 0
\end{array}\right) \quad \mathscr{J}^{5}=\left(\begin{array}{lll}
0 & 0 & 0 \\
0 & 0 & 1 \\
0 & 1 & 0
\end{array}\right) \quad \mathscr{J}^{6}=\left(\begin{array}{lll}
0 & 0 & 0 \\
0 & 0 & 0 \\
0 & 0 & 1
\end{array}\right) \text {. }
\end{aligned}
$$

Now, since $\partial \mathscr{B} / \partial B^{k}=\mathscr{J}^{k}$, and using the eigen-relations $\mathscr{B} \hat{\boldsymbol{a}}=a^{2} \hat{\boldsymbol{a}}$, etc., together with the complementary relations $\hat{\boldsymbol{a}}^{T} \mathscr{B}=a^{2} \hat{\boldsymbol{a}}^{T}$, where $\hat{\boldsymbol{a}}, \hat{\boldsymbol{b}}$ and $\hat{\boldsymbol{c}}$ are orthonormal vectors, we find

$$
\frac{\partial a^{2}}{\partial B^{k}}=\hat{\boldsymbol{a}}^{T} \mathscr{J}^{k} \hat{\boldsymbol{a}}, \quad \frac{\partial b^{2}}{\partial B^{k}}=\hat{\boldsymbol{b}}^{T} \mathscr{J}^{k} \hat{\boldsymbol{b}}, \quad \frac{\partial c^{2}}{\partial B^{k}}=\hat{\boldsymbol{c}}^{T} \mathscr{J}^{k} \hat{\boldsymbol{c}} .
$$

Combining these results with (A 3 ), we find

$$
\frac{\partial R_{F}}{\partial B^{k}}=-\frac{1}{6}\left(R_{D}\left(b^{2}, c^{2}, a^{2}\right) \hat{\boldsymbol{a}}^{T} \mathscr{J}^{k} \hat{\boldsymbol{a}}+R_{D}\left(c^{2}, a^{2}, b^{2}\right) \hat{\boldsymbol{b}}^{T} \mathscr{J}^{k} \hat{\boldsymbol{b}}+R_{D}\left(a^{2}, b^{2}, c^{2}\right) \hat{\boldsymbol{c}}^{T} \mathscr{J}^{k} \hat{\boldsymbol{c}}\right) \text {. }
$$


On the other hand, contracting $\mathscr{P}_{v}=\mathscr{M} \mathscr{D} \mathscr{M}^{T}$ with the matrices $\mathscr{J}^{k}$ gives

$$
\mathscr{P}_{v}: \mathscr{J}^{k}=\left(\mathscr{D}_{11} \hat{\boldsymbol{a}}^{T} \mathscr{J}^{k} \hat{\boldsymbol{a}}+\mathscr{D}_{22} \hat{\boldsymbol{b}}^{T} \mathscr{J}^{k} \hat{\boldsymbol{b}}+\mathscr{D}_{33} \hat{\boldsymbol{c}}^{T} \mathscr{J}^{k} \hat{\boldsymbol{c}}\right)
$$

where we have used $\mathscr{M}=(\hat{\boldsymbol{a}} \hat{\boldsymbol{b}} \hat{\boldsymbol{c}})$. Taking into account the additional factors in (A 6), (A 7) and (A 8), we may verify

$$
\mathscr{P}_{v}: \mathscr{J}^{k}=-\frac{10}{\kappa} \frac{\partial H_{v}}{\partial B^{k}}
$$

which is equivalent to (A 6).

\section{Appendix B. The focal ellipse and Maclaurin's theorem}

Laplace (1784) derived the following formula for the flow external to an ellipsoid in standard position $\left(\hat{\boldsymbol{a}}=\hat{\boldsymbol{e}}_{x}, \hat{\boldsymbol{b}}=\hat{\boldsymbol{e}}_{y}, \hat{\boldsymbol{c}}=\hat{\boldsymbol{e}}_{z}\right)$ :

$$
\psi(\boldsymbol{x})=-\frac{3 \kappa}{4} \int_{\lambda}^{\infty} \frac{\mathrm{d} u}{\sqrt{\left(u+a^{2}\right)\left(u+b^{2}\right)\left(u+c^{2}\right)}}\left(1-\frac{x^{2}}{u+a^{2}}-\frac{y^{2}}{u+b^{2}}-\frac{z^{2}}{u+c^{2}}\right),
$$

where $\lambda$ is the largest root of the cubic equation

$$
\frac{x^{2}}{\lambda+a^{2}}+\frac{y^{2}}{\lambda+b^{2}}+\frac{z^{2}}{\lambda+c^{2}}=1 \text {. }
$$

Now consider the confocal family of ellipsoids

$$
\frac{x^{2}}{\chi+a^{2}}+\frac{y^{2}}{\chi+b^{2}}+\frac{z^{2}}{\chi+c^{2}} \leqslant 1
$$

for $-a^{2} \leqslant \chi \leqslant 0$. If we substitute $u=\tilde{u}+\chi$ and $\lambda=\tilde{\lambda}+\chi$ in (B 1) and (B 2), the value of $\psi(\boldsymbol{x})$ is unaltered. If next we subtitute $\tilde{a}=\sqrt{\chi+a^{2}}, \tilde{b}=\sqrt{\chi+b^{2}}$ and $\tilde{c}=\sqrt{\chi+c^{2}}$, the value of $\psi(\boldsymbol{x})$ remains unaltered, but now we recover (B 1) and (B 2) with $a \rightarrow \tilde{a}$, $b \rightarrow \tilde{b}$, and $c \rightarrow \tilde{c}$. This proves that the entire confocal family (B 3 ) has the same flow $\psi(\boldsymbol{x})$ outside of the original ellipsoid with $\chi=0$. This result is originally due to Maclaurin (1742), see Chandrasekhar (1969).

We require $\kappa$ to be constant in (B 1). Since $\kappa=q a b c / 3$, the PV $\tilde{q}$ of a member of the confocal family must satisfy $\tilde{q}=q a b c / \tilde{a} \tilde{b} \tilde{c}$.

The limiting case $\chi \rightarrow-a^{2}$ corresponds to the focal ellipse, i.e. $\tilde{a} \rightarrow 0$. In this limit, $\tilde{q} \rightarrow \infty$, giving a sheet distribution of PV lying in the middle-major axis plane (here $x=0)$. The $P V$ density $\sigma(y, z)$ is defined by the $x$ integral of the PV across the limiting ellipsoid $\chi \rightarrow-a^{2}$, or simply the $x$ width of the ellipsoid multiplied by $\tilde{q}$ in this limit. The $x$ width $\Delta(y, z)$ is given by

$$
\begin{aligned}
\Delta & =2 \tilde{a}\left(1-\frac{y^{2}}{\tilde{b}^{2}}-\frac{z^{2}}{\tilde{c}^{2}}\right)^{1 / 2}, \\
& \rightarrow 2 \tilde{a}\left(1-\frac{y^{2}}{\tilde{\eta}^{2}}-\frac{z^{2}}{\tilde{\tau}^{2}}\right)^{1 / 2}, \\
& \equiv 2 \tilde{a} \sqrt{1-\varrho^{2}},
\end{aligned}
$$

as $\chi \rightarrow-a^{2}$, where $\eta^{2}=b^{2}-a^{2}$ and $\tau^{2}=c^{2}-a^{2}$, cf. (3.3). Hence,

$$
\begin{aligned}
\sigma & =\frac{2 q a b c}{\eta \tau} \sqrt{1-\varrho^{2}}, \\
& =\frac{6 \kappa}{\eta \tau} \sqrt{1-\varrho^{2}} .
\end{aligned}
$$




\section{Appendix C. The ellipsoidal moment model}

The 'ellipsoidal moment model' of Miyazaki et al. (2001), like the present ellipsoidal model, uses the exact expression for the self-induced flow of a vortex, but uses a moment expansion truncated to second order for vortex interactions. Here, we show that the interaction Hamiltonian $H_{i}$ found by Miyazaki et al. (2001) may be derived as a limit of the discrete form of $H_{i}$ used in the present work, i.e. (3.10).

Denote the centroid separation by $\boldsymbol{R}=\boldsymbol{X}^{\prime}-\boldsymbol{X}$, and define $\boldsymbol{r}_{i j} \equiv \boldsymbol{x}_{i}^{\prime}-\boldsymbol{x}_{j}-\boldsymbol{R}$. Note that $\boldsymbol{r}_{i j}=\left(\tilde{y}_{i}^{\prime} \eta^{\prime} \hat{\boldsymbol{b}}^{\prime}+\tilde{z}_{i}^{\prime} \tau^{\prime} \hat{\boldsymbol{c}}^{\prime}\right)-\left(\tilde{y}_{j} \eta \hat{\boldsymbol{b}}+\tilde{z}_{j} \tau \hat{\boldsymbol{c}}\right)$. Let us expand $H_{i}$ to second order in $\boldsymbol{r}_{i j} / R$, where $R=|\boldsymbol{R}|$, but use the fact that the sums over terms linear in $\boldsymbol{r}_{i j}$ vanish by symmetry (the sums over $\tilde{y}_{j}, \tilde{z}_{j}$, etc., are identically zero). The result is

$$
H_{i} \approx \check{H}_{i}=\sum_{i=1}^{n} \sum_{j=1}^{n} \kappa_{i}^{\prime} \kappa_{j}\left(\frac{1}{R}-\frac{\left|\boldsymbol{r}_{i j}\right|^{2}}{2 R^{3}}+\frac{3\left(\boldsymbol{R} \cdot \boldsymbol{r}_{i j}\right)^{2}}{2 R^{5}}\right)
$$

Next we carry out the sums, using the following results

$$
\begin{gathered}
\sum_{j=1}^{n} \kappa_{j}=1, \\
\sum_{j=1}^{n} \kappa_{j} \tilde{y}_{j}^{2}=\sum_{j=1}^{n} \kappa_{j} \tilde{z}_{j}^{2}=\frac{1}{5}, \quad \sum_{j=1}^{n} \kappa_{j} \tilde{y}_{j} \tilde{z}_{j}=0,
\end{gathered}
$$

for all orders $m>0$. Note (C2) is required to match the zeroth-order moments of the elliptical sheet, while (C 3$)$ is required to match the second-order moments. It is then straightforward to show

$$
\check{H}_{i}=\kappa^{\prime} \kappa\left(\frac{1}{R}-\frac{\eta^{\prime 2}+\tau^{\prime 2}+\eta^{2}+\tau^{2}}{10 R^{3}}+\frac{3\left[\left(\eta^{\prime} \hat{\boldsymbol{b}}^{\prime} \cdot \boldsymbol{R}\right)^{2}+\left(\tau^{\prime} \hat{\boldsymbol{c}}^{\prime} \cdot \boldsymbol{R}\right)^{2}+(\eta \hat{\boldsymbol{b}} \cdot \boldsymbol{R})^{2}+(\tau \hat{\boldsymbol{c}} \cdot \boldsymbol{R})^{2}\right]}{10 R^{5}}\right),
$$

which reduces directly to the symmetric form

$$
\begin{aligned}
\check{H}_{i}= & \kappa^{\prime} \kappa\left(\frac{1}{R}-\frac{a^{\prime 2}+b^{\prime 2}+c^{\prime 2}+a^{2}+b^{2}+c^{2}}{10 R^{3}}\right. \\
& \left.+\frac{3\left[\left(a^{\prime} \hat{\boldsymbol{a}}^{\prime} \cdot \boldsymbol{R}\right)^{2}+\left(b^{\prime} \hat{\boldsymbol{b}}^{\prime} \cdot \boldsymbol{R}\right)^{2}+\left(c^{\prime} \hat{\boldsymbol{c}}^{\prime} \cdot \boldsymbol{R}\right)^{2}+(a \hat{\boldsymbol{a}} \cdot \boldsymbol{R})^{2}+(b \hat{\boldsymbol{b}} \cdot \boldsymbol{R})^{2}+(c \hat{\boldsymbol{c}} \cdot \boldsymbol{R})^{2}\right]}{10 R^{5}}\right),
\end{aligned}
$$

independent of the order $m$. The discrete approximation is therefore not important, but simply convenient in calculating the above. This result agrees with the result obtained by Miyazaki et al. (2001), apart from notation.

The conclusion is that the moment model can be derived as an approximation to the ellipsoidal model.

\section{Appendix D. Derivatives of $H_{i}$}

To compute the flow $\boldsymbol{U}_{b}$ and $\mathscr{S}_{b}$ induced by one ellipsoid $\left\{q^{\prime}, \boldsymbol{X}^{\prime}, \mathscr{B}^{\prime}\right\}$ on another $\{q, \boldsymbol{X}, \mathscr{B}\}$, derivatives of $H_{i}$ with respect to $\boldsymbol{X}$ and $\mathscr{B}$ are required, see (2.16). The derivative with respect to $\boldsymbol{X}$ is simple, since in (3.10), $\partial \boldsymbol{x}_{j} / \partial \boldsymbol{X}=\mathscr{I}$, the identity matrix. 
Hence,

$$
\frac{\partial H_{i}}{\partial \boldsymbol{X}}=\sum_{i=1}^{n} \sum_{j=1}^{n} \kappa_{i}^{\prime} \kappa_{j} \frac{\boldsymbol{x}_{i}^{\prime}-\boldsymbol{x}_{j}}{\left|\boldsymbol{x}_{i}^{\prime}-\boldsymbol{x}_{j}\right|^{3}} .
$$

The derivative with respect to $\mathscr{B}$ is more complicated, and we follow the procedure outlined in Appendix A, taking derivatives instead with respect to each element $B^{k}$ in the representation (A 10) of $\mathscr{B}$. Since

$$
\frac{\partial H_{i}}{\partial B^{k}}=\sum_{i=1}^{n} \sum_{j=1}^{n} \kappa_{i}^{\prime} \kappa_{j} \frac{\partial \boldsymbol{x}_{j}}{\partial B^{k}} \cdot \frac{\boldsymbol{x}_{i}^{\prime}-\boldsymbol{x}_{j}}{\left|\boldsymbol{x}_{i}^{\prime}-\boldsymbol{x}_{j}\right|^{3}},
$$

we need only work out

$$
\frac{\partial \boldsymbol{x}_{j}}{\partial B^{k}}=\tilde{y}_{j}\left(\frac{\partial \eta}{\partial B^{k}} \hat{\boldsymbol{b}}+\eta \frac{\partial \hat{\boldsymbol{b}}}{\partial B^{k}}\right)+\tilde{z}_{j}\left(\frac{\partial \tau}{\partial B^{k}} \hat{\boldsymbol{c}}+\tau \frac{\partial \hat{\boldsymbol{c}}}{\partial B^{k}}\right) .
$$

First of all, using (A 12) together with $\eta=\sqrt{b^{2}-a^{2}}$ and $\tau=\sqrt{c^{2}-a^{2}}$, we have

$$
\begin{gathered}
\frac{\partial \eta}{\partial B^{k}}=\frac{1}{2 \eta}\left(\frac{\partial b^{2}}{\partial B^{k}}-\frac{\partial a^{2}}{\partial B^{k}}\right)=\frac{1}{2 \eta}\left(\hat{\boldsymbol{b}}^{T} \mathscr{J}^{k} \hat{\boldsymbol{b}}-\hat{\boldsymbol{a}}^{T} \mathscr{J}^{k} \hat{\boldsymbol{a}}\right), \\
\frac{\partial \tau}{\partial B^{k}}=\frac{1}{2 \tau}\left(\frac{\partial c^{2}}{\partial B^{k}}-\frac{\partial a^{2}}{\partial B^{k}}\right)=\frac{1}{2 \tau}\left(\hat{\boldsymbol{c}}^{T} \mathscr{J}^{k} \hat{\boldsymbol{c}}-\hat{\boldsymbol{a}}^{T} \mathscr{J}^{k} \hat{\boldsymbol{a}}\right) .
\end{gathered}
$$

The derivatives of the unit vectors, like the derivatives of the squared axis lengths, are obtained by differentiating the eigen-relations $\mathscr{B} \hat{\boldsymbol{a}}=a^{2} \hat{\boldsymbol{a}}$, etc., giving

$$
\begin{aligned}
& \mathscr{J}^{k} \hat{\boldsymbol{a}}+\mathscr{B} \frac{\partial \hat{\boldsymbol{a}}}{\partial B^{k}}=\frac{\partial a^{2}}{\partial B^{k}} \hat{\boldsymbol{a}}+a^{2} \frac{\partial \hat{\boldsymbol{a}}}{\partial B^{k}} \\
& \mathscr{J}^{k} \hat{\boldsymbol{b}}+\mathscr{B} \frac{\partial \hat{\boldsymbol{b}}}{\partial B^{k}}=\frac{\partial b^{2}}{\partial B^{k}} \hat{\boldsymbol{b}}+b^{2} \frac{\partial \hat{\boldsymbol{b}}}{\partial B^{k}} \\
& \mathscr{J}^{k} \hat{\boldsymbol{c}}+\mathscr{B} \frac{\partial \hat{\boldsymbol{c}}}{\partial B^{k}}=\frac{\partial c^{2}}{\partial B^{k}} \hat{\boldsymbol{c}}+c^{2} \frac{\partial \hat{\boldsymbol{c}}}{\partial B^{k}}
\end{aligned}
$$

Left multiplying (D 5b) by $\hat{\boldsymbol{a}}^{T}$ and $\hat{\boldsymbol{c}}^{T}$, and also (D 5c) by $\hat{\boldsymbol{a}}^{T}$ and $\hat{\boldsymbol{b}}^{T}$, we obtain

$$
\begin{array}{ll}
\hat{\boldsymbol{a}}^{T} \mathscr{J}^{k} \hat{\boldsymbol{b}}=\eta^{2} \hat{\boldsymbol{a}}^{T} \frac{\partial \hat{\boldsymbol{b}}}{\partial B^{k}}, & \hat{\boldsymbol{c}}^{T} \mathscr{J}^{k} \hat{\boldsymbol{b}}=-\xi^{2} \hat{\boldsymbol{c}}^{T} \frac{\partial \hat{\boldsymbol{b}}}{\partial B^{k}}, \\
\hat{\boldsymbol{a}}^{T} \mathscr{J}^{k} \hat{\boldsymbol{c}}=\tau^{2} \hat{\boldsymbol{a}}^{T} \frac{\partial \hat{\boldsymbol{c}}}{\partial B^{k}}, & \hat{\boldsymbol{b}}^{T} \mathscr{J}^{k} \hat{\boldsymbol{c}}=\xi^{2} \hat{\boldsymbol{b}}^{T} \frac{\partial \hat{\boldsymbol{c}}}{\partial B^{k}},
\end{array}
$$

where $\xi^{2}=c^{2}-b^{2}$. Since $\hat{\boldsymbol{a}}, \hat{\boldsymbol{b}}$ and $\hat{\boldsymbol{c}}$ are unit vectors, their derivatives are perpendicular to them. Hence, the above equations are sufficient to determine the required derivatives:

$$
\begin{aligned}
\frac{\partial \hat{\boldsymbol{b}}}{\partial B^{k}} & =\frac{1}{\eta^{2}}\left(\hat{\boldsymbol{a}}^{T} \mathscr{J}^{k} \hat{\boldsymbol{b}}\right) \hat{\boldsymbol{a}}-\frac{1}{\xi^{2}}\left(\hat{\boldsymbol{c}}^{T} \mathscr{J}^{k} \hat{\boldsymbol{b}}\right) \hat{\boldsymbol{c}}, \\
\frac{\partial \hat{\boldsymbol{c}}}{\partial B^{k}} & =\frac{1}{\tau^{2}}\left(\hat{\boldsymbol{a}}^{T} \mathscr{J}^{k} \hat{\boldsymbol{c}}\right) \hat{\boldsymbol{a}}+\frac{1}{\xi^{2}}\left(\hat{\boldsymbol{b}}^{T} \mathscr{J}^{k} \hat{\boldsymbol{c}}\right) \hat{\boldsymbol{b}} .
\end{aligned}
$$

Note that $\hat{\boldsymbol{a}}^{T} \mathscr{J}^{k} \hat{\boldsymbol{b}}=\hat{\boldsymbol{b}}^{T} \mathscr{J}^{k} \hat{\boldsymbol{a}}$, etc., are simple scalars, e.g. $\hat{\boldsymbol{a}}^{T} \mathscr{J}^{1} \hat{\boldsymbol{b}}=\hat{a}_{1} \hat{b}_{1}, \hat{\boldsymbol{a}}^{T} \mathscr{J}^{2} \hat{\boldsymbol{b}}=$ $\hat{a}_{1} \hat{b}_{2}+\hat{a}_{2} \hat{b}_{1}$, and so forth. 


\section{REFERENCES}

Chandrasekhar, S. 1969 Ellipsoidal Figures of Equilibrium. Dover, New York, 255 pp.

Dritschel, D. G. 2002 Vortex merger in rotating stratified flows. J. Fluid Mech. 455, 83-101.

Dritschel, D. G. \& Legras, B. 1991 The elliptical model of two-dimensional vortex dynamics. Part II: disturbance equations. Phys. Fluids A 3, 855-868.

Dritschel, D. G. \& ViúdeZ, A. 2003 A balanced approach to modelling rotating stably-stratified flows. J. Fluid Mech. 488, 123-150.

Gill, A. E. 1982 Atmosphere-Ocean Dynamics. Academic, 662 pp.

Hashimoto, H., Shimonishi, T. \& Miyazaki, T. 1999 Quasigeostrophic ellipsoidal vortices in a two-dimensional strain field. J. Phys. Soc. Japan 68, 3863-3880.

Holton, J. R. 1982 An Introduction to Dynamic Meteorology, 3rd edn. Academic, 511 pp.

Hoskins, B. J., McIntyre, M. E. \& Robertson, A. W. 1985 On the use and significance of isentropic potential-vorticity maps. Q. J. R. Met. Soc. 111, 877-946.

KIDA, S. 1981 Motion of an elliptical vortex in a uniform shear flow. J. Phys. Soc. Japan 50, $3517-3880$.

Khavinson, D. \& Shapiro, H. S. 1989 The Schwarz potential in $R^{N}$ and Cauchy's problem for the Laplace equation. Unpublished report TRITA-MAT-1989-36, Royal Institute of Technology, Sweden.

Laplace, P. S. 1784 Théorie du Mouvement et de la Figure Elliptique des Planètes. Imprimerie de Ph.-D. Pierres, Paris, 153 pp.

Legras, B. \& Dritschel, D. G. 1991 The elliptical model of two-dimensional vortex dynamics. Part I: the basic state. Phys. Fluids A 3, 845-854.

McKiver, W. J. \& Dritschel, D. G. 2003 The motion of a fluid ellipsoid in a general linear background flow. J. Fluid Mech. 474, 147-173.

Maclaurin, C. 1742 A Treatise on Fluxions. Printed by T. W. and T. Ruddimans, Edinburgh, 2 vols, $763 \mathrm{pp}$.

McWilliams, J. C., Weiss, J. B. \& Yavneh, I. 1999 The vortices of homogeneous geostrophic turbulence. J. Fluid Mech. 401, 1-26.

Meacham, S. P. 1992 Quasigeostrophic, ellipsoidal vortices in stratified fluid. Dyn. Atmos. Oceans 16, 189-223.

Meacham, S. P., Morrison, P. J. \& Flierl, G. R. 1997 Hamiltonian moment reduction for describing vortices in shear. Phys. Fluids 9, 2310-2328.

Meacham, S. P., Pankratov, K. K., Shchepetkin, A. F. \& Zhmur, V. V. 1994 The interaction of ellipsoidal vortices with background shear flows in a stratified fluid. Dyn. Atmos. Oceans 21, $167-212$.

Melander, M. V., Zabusky, N. J. \& Styczek, A. S. 1986 A moment model for vortex interactions of the two-dimensional Euler equations. 1. Computational validation of a Hamiltonian elliptic representation. J. Fluid Mech. 167, 95-115.

Miyazaki, T., Furuichi, Y. \& TAKahashi, N. 2001 Quasigeostrophic ellipsoidal vortex model. J. Phys. Soc. Japan 70, 1942-1953.

Miyazaki, T., Ueno, K. \& Shimonishi, T. 1999 Quasigeostrophic tilted spheroidal vortices. J. Phys. Soc. Japan 68, 2592-2601.

ReINAUd, J. N. \& Dritschel, D. G. 2002 The merger of vertically offset quasi-geostrophic vortices. J. Fluid Mech. 469, 287-315.

Reinaud, J. N. \& Dritschel, D. G. 2003 The critical merger distance between two co-rotating quasigeostrophic vortices. J. Fluid Mech. submitted. Also available at: http://www-vortex.mcs.stand.ac.uk/ dgd/ELM.

Simmons, A. J. \& Hoskins, B. J. 1976 Baroclinic instability on the sphere: normal modes of the primitive and quasi-geostrophic equations. J. Atmos. Sci. 33, 1454-1477.

Zhmur, V. V. \& Pankratov, K. K. 1990 Distant interaction of an ensemble of quasigeostrophic ellipsoidal eddies: Hamiltonian formulation. Izv. Akad. Nauk. SSSR Phys. Atmos. Ocean. 26, 972-981.

Zhmur, V. V. \& Shchepetkin, A. F. 1991 Evolution of an ellipsoidal vortex in a stratified ocean: survivability of the vortex in flow with vertical shear. Izv. Akad. Nauk. SSSR Phys. Atmos. Ocean. 27, 492-503. 